\title{
Evx1 and Evx2 specify excitatory neurotransmitter fates and suppress inhibitory fates through a Pax2- independent mechanism
}

\author{
José L. Juárez-Morales ${ }^{1}$, Claus J. Schulte², Sofia A. Pezoa', Grace K. Vallejo' ${ }^{1}$ William C. Hilinski ${ }^{1,3}$,
} Samantha J. England', Sarah de Jager ${ }^{2}$ and Katharine E. Lewis ${ }^{1 *}$ (i)

\begin{abstract}
Background: For neurons to function correctly in neuronal circuitry they must utilize appropriate neurotransmitters. However, even though neurotransmitter specificity is one of the most important and defining properties of a neuron we still do not fully understand how neurotransmitter fates are specified during development. Most neuronal properties are determined by the transcription factors that neurons express as they start to differentiate. While we know a few transcription factors that specify the neurotransmitter fates of particular neurons, there are still many spinal neurons for which the transcription factors specifying this critical phenotype are unknown. Strikingly, all of the transcription factors that have been identified so far as specifying inhibitory fates in the spinal cord act through Pax2. Even Tlx1 and Tlx3, which specify the excitatory fates of $\mathrm{dl} 3$ and $\mathrm{dl} 5$ spinal neurons work at least in part by down-regulating Pax2.
\end{abstract}

Methods: In this paper we use single and double mutant zebrafish embryos to identify the spinal cord functions of Evx1 and Evx2.

Results: We demonstrate that Evx1 and Evx2 are expressed by spinal cord VOV cells and we show that these cells develop into excitatory (glutamatergic) Commissural Ascending (CoSA) interneurons. In the absence of both Evx 1 and Evx2, Vov cells still form and develop a CoSA morphology. However, they lose their excitatory fate and instead express markers of a glycinergic fate. Interestingly, they do not express Pax2, suggesting that they are acquiring their inhibitory fate through a novel Pax2-independent mechanism.

Conclusions: Evx1 and Evx2 are required, partially redundantly, for spinal cord VOv cells to become excitatory (glutamatergic) interneurons. These results significantly increase our understanding of the mechanisms of neuronal specification and the genetic networks involved in these processes.

Keywords: Spinal cord, Interneuron, Zebrafish, Evx, Pax2, Glutamatergic, Neurotransmitter, CNS, Transcription factor, V0

\section{Background}

Hundreds of millions of people across the world are affected by neurological diseases and injuries. Understanding how functional neuronal circuitry is established in the vertebrate central nervous system (CNS) is essential for developing better treatments for these conditions. How neuronal circuitry develops is also a fundamental

\footnotetext{
* Correspondence: kelewi02@syr.edu

'Department of Biology, Syracuse University, 107 College Place, Syracuse, NY 13244, USA

Full list of author information is available at the end of the article
}

question in developmental neuroscience. To answer this question, we need to identify how the functional properties of distinct neurons are specified; since these properties determine which circuits the neurons participate in, the functional roles that the neurons have within those circuits and the resulting outputs of the circuitry. The spinal cord is a powerful system for establishing fundamental principles of neuronal fate specification, function and circuit assembly, as it is relatively simple and experimentally tractable compared to the brain. This has enabled considerable progress in establishing the functions 
of different ventral spinal cord interneurons in locomotor circuitry (e.g. [1-9]). However, we still know relatively little about how the functional properties of these cells are determined.

For neurons to function correctly they must synthesize and utilize correct neurotransmitters. Within neuronal circuitry, if they inhibit rather than excite their synaptic partners, or vice versa, then the behaviors and functional outputs of those circuits will be dramatically disturbed, and may give rise to pathological conditions. For example, disruptions in the balance of excitatory and inhibitory neurons in the CNS have been implicated in epilepsy, autism, Alzheimer's and many other neurological disorders (e.g. [10-13]). However, even though neurotransmitter specificity is one of the most important and defining properties of a neuron we still do not fully understand how neurotransmitter fates are specified during development.

Many neuronal properties are determined by the transcription factors that cells express as they start to differentiate. We already know a few transcription factors (e.g. Ptf1a, Lhx1, Lhx5, Lbx1, Pax2) that specify the inhibitory (GABAergic and/or glycinergic) fates of several subsets of spinal interneurons [14-18]. Strikingly, most of these transcription factors function in dorsal spinal neurons and all of them act through Pax2 [14-21]. In contrast, we only know two transcription factors, Tlx1 and Tlx3, that are required for the specification of excitatory (glutamatergic) fates and these are only expressed in dorsal dI3, dI5 and $\mathrm{DIL}_{\mathrm{B}}$ cells $[15,16,22]$. Interestingly, Tlx1 and Tlx3 determine the glutamatergic fates of dI3 and dI5 cells at least in part by downregulating Pax2 [15]. These results suggest that Pax2 is a crucial player in neurotransmitter fate specification with its presence being required for inhibitory fates and its absence required for excitatory fates. However, we still do not know which transcription factors regulate the neurotransmitter fates of many excitatory spinal neurons, including those in the ventral spinal cord, whose correct functional specification is essential for locomotion.

In this paper we identify two transcription factors, Evx1 and Evx2, which are required for a subset of excitatory fates in the ventral spinal cord. In mammals, the spinal cord expression of Evx1 and Evx2 is restricted to a population of cells located in an intermediate dorso-ventral position corresponding to V0 cells (e.g. [23-28]). V0 cells are post-mitotic cells that form from the p0 (Dbx1-positive, Nkx6.2-negative) progenitor domain [23, 27-29]. These cells develop into interneurons that are important components of locomotor circuitry and they can be subdivided into an Evx1-positive sub-population called $\mathrm{VO}_{\mathrm{v}}$ cells and an Evx1-negative sub-population called $\mathrm{VO}_{\mathrm{D}}$ cells. These names reflect the fact that V0v cells form more ventrally than $\mathrm{V}_{\mathrm{D}}$ cells (e.g. [23-28, 30-34]). Evx2 is expressed in a similar pattern to Evx1 in the mouse CNS, suggesting that it may also be expressed by $\mathrm{VOv}$ cells. This is consistent with the observation that Evx2 spinal cord expression is lost in mouse Evx1 mutants [23]. However, co-expression of Evx1 and Evx2 in the mouse spinal cord has not yet been demonstrated [24].

In mammals, both $\mathrm{V} 0 \mathrm{v}$ and $\mathrm{V}_{\mathrm{D}}$ interneurons are crucial for correct left-right alternation during locomotion, with V0v cells in particular being required for hindlimb left-right alternation during fast locomotion $[9,34]$. While the functions of V0 cells in specific behaviors have so far only been assayed in mouse, these cells have highly conserved commissural axon trajectories in all animals examined so far $([23-28,32,33,35,36]$; this paper), suggesting that their functional properties are likely to be highly conserved across the vertebrate lineage. However, when we started this work the neurotransmitter phenotype of V0v cells had not been identified.

In zebrafish, evx1 and evx2 are expressed in a similar intermediate dorsal-ventral spinal cord position to that observed in other vertebrates [26, 32, 33], although again, co-expression of these two genes has not previously been demonstrated. In this paper, we confirm that $e v x 1$ and $e v x 2$ are co-expressed by $\mathrm{V} 0 \mathrm{v}$ cells and we show that V0v cells are glutamatergic and have a Commissural Ascending (Comissural Secondary Ascending or CoSA) morphology. We also provide the first analysis of evx1;evx2 double mutants in any vertebrate and the first analysis of the spinal cord phenotype of $e v \times 2 \mathrm{mu}-$ tants. Significantly, we demonstrate that Evx1 and Evx2 are required, partially redundantly, to specify the glutamatergic fates of V0v cells. Given that we know so little about how excitatory fates are specified in the spinal cord and particularly the ventral spinal cord, these findings add considerably to our understanding of CNS circuit development.

In the absence of both Evx1 and Evx2, V0v cells lose their glutamatergic fates but other functional characteristics like soma/cell body morphology and axon trajectory are unchanged. In addition, and in contrast to a previously described mouse Evx1 mutant [23], these cells do not express markers of neighboring cell types. This suggests that V0v cells are not transfating into a different class of neuron; they have just changed some of their functional properties. Strikingly, in evx1;evx2 double mutants V0v cells become inhibitory, but they do not express Pax2, suggesting that they are acquiring their inhibitory fates through a novel Pax2-independent mechanism. 


\section{Methods}

\section{Ethics approval}

All zebrafish experiments in this research were approved either by the UK Home Office or by the Syracuse University IACUC committee.

\section{Zebrafish husbandry and fish lines}

Zebrafish (Danio rerio) were maintained on a $14 \mathrm{~h}$ light/ $10 \mathrm{~h}$ dark cycle at $28.5{ }^{\circ} \mathrm{C}$ and embryos were obtained from natural, paired and/or grouped spawnings of wildtype (WT) adults (AB, TL or AB/TL hybrids), identified heterozygous or homozygous $\mathrm{Tg}$ (slc17a6:EGFP) (used to be called $\operatorname{Tg}(v G l u t 2 a: E G F P) ;[36,37]), \operatorname{Tg}(\text { evx1:EGFP })^{S U 1}$ or $\operatorname{Tg}(\text { evx1:EGFP })^{S U 2}$ adults, double heterozygous $e v x 1^{i 232}$;evx $2^{\text {sa140 }}$ mutants or double heterozygous evx $1^{i 232}$;evx $2^{\text {sa140 }}$ mutants that also carried one of the $T g$ (evx1:EGFP) lines (see below). Embryos were reared at $28.5^{\circ} \mathrm{C}$ and staged by hours post fertilization (h), days post or prim staging/or prim staging [38].

The evx1 $1^{i 232}$ mutation has been described before [39]. The $e v x 2^{\text {sa140 }}$ mutant was received from the Wellcome Trust Sanger Centre, (https://www.sanger.ac.uk/sanger/ Zebrafish_Zmpbrowse). Both mutations produce a single base pair change (a $\mathrm{C}$ to a $\mathrm{T}$ in the case of $e v x 2^{\mathrm{sa140}}$ ) that results in a premature stop codon before the homeobox ([39]; Fig. 1a). Therefore, the truncated proteins, if formed, will lack DNA binding domains. The evx $2^{\text {sa140 }}$ mutation creates a $B f a I$ recognition site that enables us to genotype individual fish and embryos (see below; Fig. 1b). In this paper we demonstrate that the $e v x 2^{\text {sal } 140}$ allele does not make Evx2 protein (Fig. 1c), strongly sug-

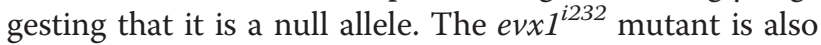
probably a null allele [39]. However, in contrast to the evx $1^{i 232}$ mutant which is viable, the $e v x 2^{\text {sal } 140}$ mutant is embryonic lethal (see Results and Additional file 1: Results).

\section{Genotyping}

Genotyping of mutant alleles was performed on both live adults and fixed embryos using DNA extracted from fin clips and dissected heads respectively. Fin clipping and evx1 genotyping were performed as in [39]. To extract DNA from embryos, heads were removed in $80 \%$ glycerol / $20 \%$ PBS with insect pins. Embryonic trunks were stored in $70 \%$ glycerol / $30 \%$ PBS at $4{ }^{\circ} \mathrm{C}$ for later analysis. Heads were incubated in $50 \mu \mathrm{L}$ of Proteinase $\mathrm{K}$ buffer solution (1 M Tris- $\mathrm{HCl}, \mathrm{pH} 8.2 ; 0.5 \mathrm{M}$ EDTA; $1 \mathrm{M} \mathrm{NaCl} ; 20 \%$ SDS; $10 \mathrm{mg} / \mathrm{ml}$ Proteinase K) for $2 \mathrm{~h}$ at $55{ }^{\circ} \mathrm{C}$. Proteinase $\mathrm{K}$ was heat inactivated at $100{ }^{\circ} \mathrm{C}$ for $10 \mathrm{~min}$ and tubes were centrifuged for $20 \mathrm{~min}$ at $13,000 \mathrm{rpm}$. DNA was precipitated with $100 \%$ ethanol at $-20{ }^{\circ} \mathrm{C}$ overnight, centrifuged to pellet the DNA and re-suspended in $20 \mu \mathrm{L}$ of water. $2 \mu \mathrm{L}$ of DNA was used for each PCR.
The $e v x 2^{s a 140}$ mutation creates a $B f a I$ recognition site. A genomic region flanking the mutation site was PCR amplified using the following conditions: $94{ }^{\circ} \mathrm{C}$ for $60 \mathrm{~s}$, followed by 5 cycles of $92{ }^{\circ} \mathrm{C}$ for $30 \mathrm{~s}, 54{ }^{\circ} \mathrm{C}$ for $30 \mathrm{~s}, 72{ }^{\circ} \mathrm{C}$ for $60 \mathrm{~s}$; followed by 40 cycles of $92{ }^{\circ} \mathrm{C}$ for $20 \mathrm{~s}, 52{ }^{\circ} \mathrm{C}$ for $30 \mathrm{~s}$, and $72{ }^{\circ} \mathrm{C}$ for $60 \mathrm{~s}$, followed by a final extension at $72{ }^{\circ} \mathrm{C}$ for 5 min. Forward primer: GTAATGCGATCCCAAAACG. Reverse primer: TTATTTTAGATTTGGCAATGG.

PCR products were digested with $B f a I$ and analysed on a $1 \%$ agarose gel. The WT product is $454 \mathrm{bp}$, whereas the mutant product is cut into $218 \mathrm{bp}$ and $236 \mathrm{bp}$ fragments. These fragments are close enough in size that they are usually detected as one band on an agarose electrophoresis gel (Fig. 1b).

\section{Creation of $T g(e v \times 1: E G F P)$ lines}

Potential evx1 enhancer regions were identified by multispecies sequence comparisons using the global alignment program Shuffle-LAGAN [40] and visualized using VISTA [41]. Zebrafish (Danio rerio) evx1 genomic sequence (ENSDARG00000099365) and orthologous sequences from human (ENSG00000106038) and mouse (ENSMUSG00000005503) were obtained from Ensembl (http://www.ensembl.org). Zebrafish sequence was used as the baseline and annotated using exon/intron information from Ensembl. The alignment was performed using a $100 \mathrm{bp}$ window and a cutoff score of $70 \%$ identity. A multi-species comparison of approximately $23 \mathrm{~Kb}$ of Danio rerio genomic DNA sequence containing evx1 and extending $5 \mathrm{~Kb}$ into flanking regions revealed high conservation in both coding and non-coding sequences among compared species. We identified three Conserved Non-coding Elements (CNEs) located 5' and 3' to evx1. The first is located $79 \mathrm{bp}$ upstream of zebrafish evx1 coding sequence and extends over $100 \mathrm{bp}$. The other two are located 3' to evx1. One is $2354 \mathrm{bp}$ downstream of the stop codon and is 184 bp long whereas the other is $2979 \mathrm{bp}$ downstream of the stop codon and extends over 140 bp (Fig. 1d). One amplicon encompassing these two 3' CNEs and the intervening sequence $(1.34 \mathrm{~Kb})$ was PCR-amplified from genomic DNA. Forward primer: AAGATTGGAATGGAATGTCT. Reverse primer: GCA TTTTCGCCTTTGCATCA.

The $\operatorname{Tg}(e v x 1: E G F P)^{S U 1}$ line was generated by cloning this 3' CNE amplicon into the pDONR ${ }^{\mathrm{TM}} \mathrm{P} 4-\mathrm{P} 1 \mathrm{R}$ vector from Invitrogen using Gateway technology [42, 43]. The final construct was assembled using the pENTRbasegfp plasmid and the pCSDest2 vector [44]. This resulted in a vector containing Tol2:1.3Kb 3' zfish evx1:ßcarp minimal promoter:EGFP:Tol2.

The same 3' CNE amplicon was used to generate the $\operatorname{Tg}(e v x 1: E G F P)^{S U 2}$ line. In this case, the GAL4VP16;UASEGFP cassette was taken from the pBGAL4VP16;UASEGFP plasmid [45] and cloned into a middle entry vector 


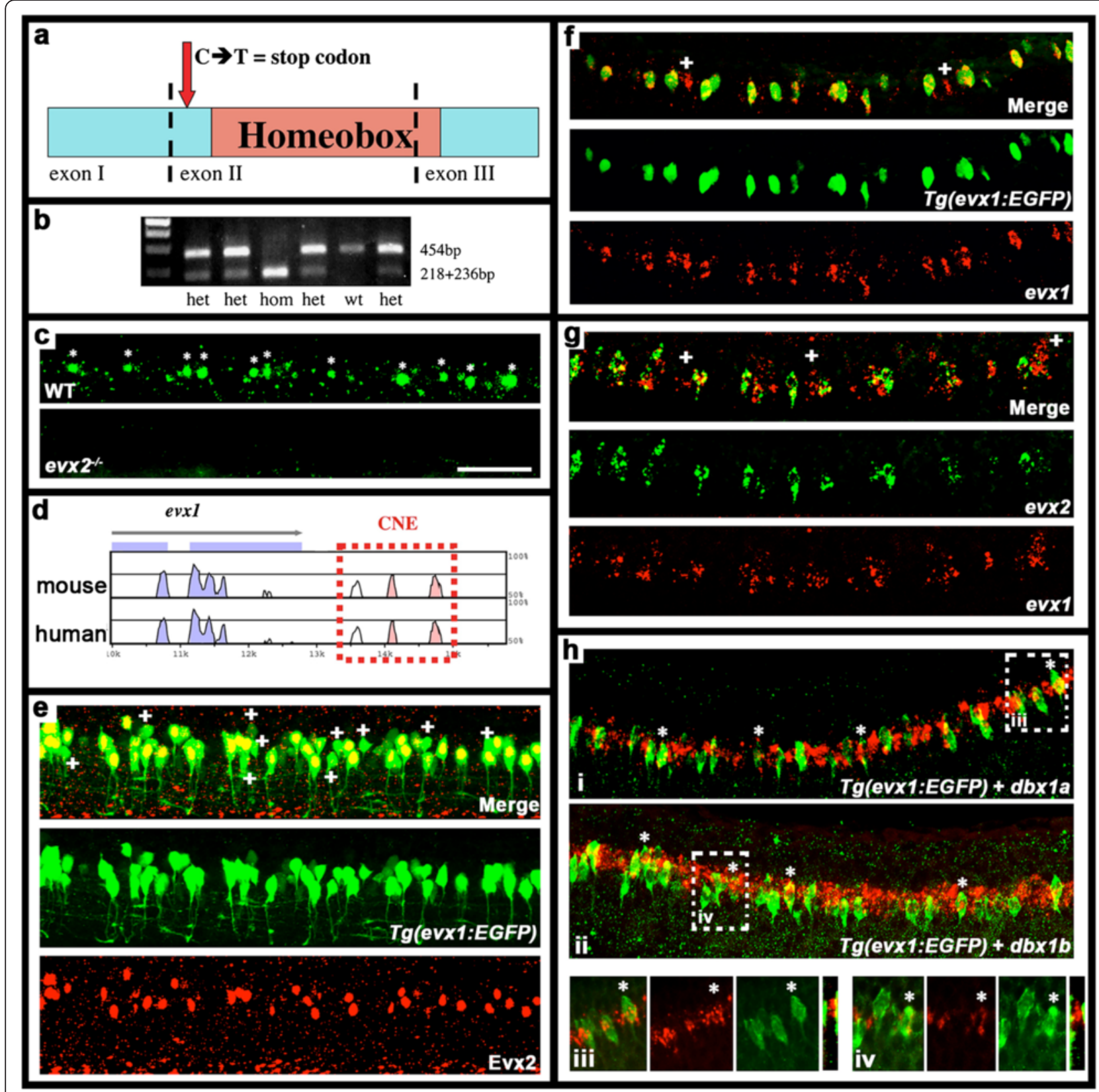

Fig. 1 Vov cells co-express evx1 and evx2. a Schematic of Evx2 showing exon boundaries (dotted lines), homeobox-domain (pink) and location of evx $2^{\text {sal40 }}$ mutation (red arrow). b Examples of genotyping WT, heterozygous and homozygous evx $2^{\text {sal } 140}$ mutant embryos using gel electrophoresis (see methods). The two fragments from the mutant allele restriction product run at the same position on the gel. c, e-h lateral views, dorsal up, anterior left, of spinal cord at $24 \mathrm{~h}$ (f-h) or $27 \mathrm{~h}$ (c \& e). c Evx2 immunohistochemistry on WT (top panel) and homozygous evx2 $2^{\text {sal } 40}$ mutant (bottom panel) embryos. Stars indicate Evx2-expressing cells. Mutant embryos have no Evx2 expression. d Schematic showing Shuffle-LAGAN analysis of evx1 genomic region with zebrafish sequence as baseline compared to orthologous regions in mouse and human genomes. Conserved coding sequences are indicated in blue, arrow indicates 5'-3' orientation. CNEs in 3' region are indicated in pink. The region amplified to create transgenic lines is indicated with red dotted lines. e-h Double staining for (e) EGFP (green) and Evx2 (red) in Tg(evx1:EGFP) ${ }^{\mathrm{SU} /}$ embryos, (f) EGFP (green) and evx1 (red) in Tg(evx1:EGFP) $)^{\mathrm{SU} 1}$ embryos, (g) evx1 (red) and evx2 (green) in WT embryos, (h) EGFP (green) and dbx1a (red) in Tg(evx1:EGFP) ${ }^{\text {SU1 }}$ embryos (i and iii) and EGFP (green) and $d b \times 1 b$ (red) in Tg(evx1:EGFP) ${ }^{S U 1}$ embryos (ii and iv). In e-g and $\mathbf{h} \_$iii - $\mathbf{h} \_$iv, merged and single channel views are provided. White crosses indicate cells that only express evx1. In (f) these probably represent cells that have just started to express evx 1 as there is a delay in expression of EGFP. White stars in $\mathbf{h}$ indicate double-labelled cells. Three wider panels at bottom of $\mathbf{h}$ (iii and iv) are magnified single-confocal-plane views of white dotted rectangle regions in panels $\mathbf{h} \_i$ and $\mathbf{h} \_$ii respectively. Thin panel on RHS in each case shows a cross-section projection (slice) created in Image J confirming that GFP expression is lateral to $d b x$ expression. Scale bar: $50 \mu \mathrm{m}$ (c \& e-h) 
from Invitrogen [42, 43]. An oligonucleotide containing the cfos minimal promoter sequence [46] plus $17 \mathrm{bp}$ of the 5' arm of GAL4 was synthesized and used with a RVeGFPAttb2 primer to PCR amplify the GAL4VP16;UAS-EGFP cassette.

FWcfosGAL4VP16 primer: CACTCATTCATAAAAC GCTTGTTATAAAAGCAGTGGCTGCGGCGCCTCG TACTCCAACCGCATCTGCAGCGAGCAACTGAGA AGCCAAGACTGAGCCGGCGGCCTTTGTACAAAA AAGCAG

RVeGFPAttb2 primer: GGGGACCACTTTGTACAAG AAAGCTGGGTTTACTTGTACAGCTCGTCCA.

This PCR product was used to generate a second PCR product using the primer FWattB1cfos: GGGGACAAG TTTGTACAAAAAAGCAGGCTCACTCATTCATAAA ATCGCTT and the RVeGFPAttb2 primer.

The final amplicon was cloned into $\mathrm{pDONR}^{\mathrm{m} \mathrm{m}} 221$ using gateway technology. This middle entry vector was used to generate a final vector containing Tol2:1.3Kb 3' zfish evx1:cfos minimal promoter:GAL4VP16;UAS-EGFP:Tol2.

Each of these plasmids was separately co-injected with transposase mRNA into 1-2 cell embryos as described by [47]. Embryos were raised to adulthood and outcrossed to identify founders. In each case one stable transgenic line was generated. The $\operatorname{Tg}(\operatorname{evx} 1: E G F P)^{S U 2}$ or $\operatorname{Tg}(1.3 \mathrm{~kb}$ evx1:cfos:GAL4-UAS:EGFP) line has the advantage that it contains a GAL4-UAS cassette to amplify EGFP expression. This facilitates visualization of axons. However, this line has a slightly more variegated expression than the $\operatorname{Tg}(\text { evx } 1: E G F P)^{S U 1}$ or $T g(1.3 \mathrm{~Kb}$ evx1:ßcarp:EGFP) line, presumably because of stochastic silencing of the construct due to the GAL4-UAS sequences [48]. In contrast the $\operatorname{Tg}(e v x 1: E G F P)^{S U 1}$ line labels all V0v cells more consistently, but the EGFP expression is slightly weaker and we were never able to obtain $e v x 1^{-/-} ; e v x 2^{-/-}$double mutant embryos that contained this transgene, even though we could obtain $e v x 1^{-/-} ; e v x 2^{+/+}$and $e v x 1^{-/-} ; e v x 2^{+/-}$embryos. This suggests that the $T g(1.3 \mathrm{~Kb}$ evx $1: \beta c a r p: E G F P)$ construct integrated close to the WT evx2 allele.

\section{Morpholino injection}

Approximately $5 \mathrm{nl}$ of a 1:1 combination of two Evx2 ATG Morpholino antisense oligonucleotides (MOs) at $1.25 \mathrm{mg} / \mathrm{ml}$ each were injected into $1-2$ cell embryos (evx2-1 MO: TTCTTTTCTTATCCTCTCCATCATG; evx2-2 MO: AATCCAAAGTCCCAGGGCTGGTGCT). In all cases, we confirmed that the MOs had completely knocked down Evx2 using immunohistochemistry for zebrafish Evx2.

\section{Expression profiling Vov cells}

To determine which neurotransmitters V0v cells express and to identify additional transcription factors expressed by these cells, different combinations of spinal cord and trunk cells were extracted from live transgenic zebrafish embryos at $27 \mathrm{~h}$ using fluorescence activated cell-sorting (FACS). Prior to FACS, embryos were prim-staged, deyolked, dissected and dissociated as in $[49,50]$. In all cases, the heads were removed to ensure that only trunk or spinal cord cells were collected. Pure populations of cells were obtained using combinations of the following transgenic lines: $\operatorname{Tg}\left(\right.$ elav13:EGFP), $\operatorname{Tg}(\text { evx1:EGFP })^{\text {SUI }}$, $\operatorname{Tg}($ pax2a:GFP), $\quad \operatorname{Tg}(X l a . T u b b: D s R e d \quad$ (formerly $\operatorname{Tg}($ NBT:DsRed)), $\operatorname{Tg}(v s \times 2: D s R e d)$ and $T g$ (gata1:GFP) [8, 51-54]. Trunk samples correspond to FAC-sorted trunk cells (spinal cord and other tissues). All neuron samples are EGFP-positive cells from $\mathrm{Tg}$ (elav13:EGFP) trunks. V0v neurons are EGFP-positive cells from $\mathrm{Tg}(\mathrm{ev}$ $x 1: E G F P)^{S U 1}$ trunks. V1 neurons are double-positive EGFP-positive, DsRed-positive cells from $\operatorname{Tg}$ (pax2a:GFP); $\operatorname{Tg}(X l a . T u b b: D s R e d)$ trunks. V2a neurons are double-positive DsRed-positive, EGFP-positive cells from $\operatorname{Tg}(v s \times 2: D s R e d) ; \operatorname{Tg}($ elavl3:EGFP) trunks. V2b + KA neurons are double-positive EGFP-positive, DsRed-positive cells from $T g$ (gata1:GFP); $\operatorname{Tg}(X l a . T u b b: D s R e d)$ trunks. Total RNA was extracted using an RNeasy Micro Kit (Qiagen, 74004). RNA quality and quantity was assayed on an Agilent 2100 Bioanalyser (RNA 6000 Pico Kit, Agilent, 5067-1513), before converting to fluorescentlylabelled cDNA (Ovation Pico WTA System V2, Pico, 3302) and hybridizing to a custom-designed Agilent microarray (EMBL Genomics Core, Heidelberg). Details of this microarray will be described elsewhere, along with the characterization of additional genes identified from these analyses. Data pre-processing and normalization was performed using Bioconductor software (https:// www.bioconductor.org/). Two-class eBayes and threeclass ANOVA analyses were performed using GEPAS software (Tárraga, (2008)). All reported statistics were corrected for multiple testing (Benjamini and Hochberg (1995)).

\section{in situ hybridization}

Embryos were fixed in $4 \%$ paraformaldehyde and single and double in situ hybridizations were performed as previously described $[55,56]$. RNA probes were prepared using the following templates, $d b \times 1 a$ and $d b \times 1 b$ [57], evx1 [58], evx2 [32], eve1 [59], pax2a, pax2b, pax8 [60] and eng1b [14]. To determine neurotransmitter phenotypes we used probes for genes that encode proteins that transport or synthesize specific neurotransmitters. A mixture of two probes (glyt $2 a$ and glyt $2 b$ ) for slc6a5 (previously called glyt2) was used to label glycinergic cells [61, 62]. slc6a5 encodes for a glycine transporter necessary for glycine reuptake and transport across the plasma membrane. A mixture of two probes to gad1b 
(previously called gad67, probes used to be called gad67a and gad67b) and one probe to gad2 (previously called gad65) was used to label GABAergic cells [61, 62]. gad1b and gad2 encode for glutamic acid decarboxylases, necessary for the synthesis of GABA from glutamate. A mixture of slc17a6b (formerly called vglut2.1) and slc17a6a (formerly called vglut 2.2) probes was used to label glutamatergic cells [61, 62]. These genes encode proteins responsible for transporting glutamate to the synapse. In all of these cases, a mix of equal concentrations of the relevant probes was used [61, 62]. We also used slc32a1 (formerly called viaat), which encodes for a vesicular inhibitory amino acid transporter, to label all inhibitory cells [8].

The DNA template for the skor2 (ZDB-GENE-06082557) probe was generated by PCR-amplifying the 3 ' region of skor 2 from cDNA using a reverse primer containing a T3 promoter sequence at the 5 ' end (indicated in italics below). Total RNA was extracted by homogenizing 50$100 \mathrm{mg}$ of 27hpf wild-type zebrafish embryos in $1 \mathrm{~mL}$ of TRIzol reagent (Ambion, 15596-026). cDNA was synthesized using Bio-Rad iScript Reverse Transcription Supermix kit (Bio-Rad, 170-8891). A $50 \mu \mathrm{L}$ PCR was assembled containing $5 \mu \mathrm{L}$ cDNA and 1 unit of Phusion High-Fidelity DNA Polymerase (NEB, M0530L). PCR conditions were: $94{ }^{\circ} \mathrm{C}$ for $3 \mathrm{~min}$ followed by 35 cycles of $94{ }^{\circ} \mathrm{C}$ for $30 \mathrm{~s}, 56.5{ }^{\circ} \mathrm{C}$ for $30 \mathrm{~s}, 72{ }^{\circ} \mathrm{C}$ for $1.5 \mathrm{~min}$ and then a final extension step of $72{ }^{\circ} \mathrm{C}$ for $10 \mathrm{~min}$. PCR product was purified by phenol:chloroform extraction. Forward primer: CGCAAGACGCTTTTTATCC

Reverse primer: AATTAACCCTCACTAAAGGGAAAA TGGAGAGCTGCCTTTCAG.

ZFIN Identification numbers are provided for all genes in Additional file 1: Table S2.

\section{Immunohistochemistry}

Primary antibodies used were rabbit anti-Evx2 (a kind gift from Dr Higashijima, described in Satou et al., 2012, raised against the first 168 amino acids of zebrafish Evx2, a region with no significant homology to zebrafish Evx1, 1:300), mouse anti-GFP (Roche Applied Science, 11814460001, 1:500), rabbit anti-GFP (Molecular Probes A6465, 1:500) and mouse anti-Pax2 (Covance PRB-276P 1:300). The Pax2 antibody recognizes both Pax2a and Pax2b in zebrafish [14]. Antibodies used for fluorescent in situ hybridization were mouse anti-Dig (Jackson ImmunoResearch 200-002-156, 1:5000) and rabbit anti-Flu (Invitrogen A889, 1:2500). These were detected with Invitrogen Tyramide kits \#12 and \#5. Secondary antibodies used were Alexa Fluor 568 goat anti-rabbit (Molecular Probes A11036, 1:500), Alexa Fluor 488 goat anti-rabbit (Molecular Probes A11034, 1:500) and Alexa Fluor 488 goat anti-mouse (Molecular Probes A11029, 1:500).
Embryos for immunohistochemistry were treated with acetone for $15 \mathrm{~min}$ (24 h embryos) or $20 \mathrm{~min}(30 \mathrm{~h}$ embryos) to permeabilize them, washed for $5 \mathrm{~min}$ in distilled water, then washed $2 \times 10 \mathrm{~min}$ in PBS. Embryos were treated with Image-iT Signal Enhancer (Invitrogen, I36933) for $30 \mathrm{~min}$, then incubated in block solution (2\% goat serum, $1 \%$ BSA, $10 \%$ DMSO and $0.5 \%$ Triton) for $1 \mathrm{~h}$ at room temperature followed by incubation in primary antibody in fresh block solution at $4{ }^{\circ} \mathrm{C}$ overnight. Embryos were washed with PBT (PBS $+0.1 \%$ Triton) for $2 \mathrm{~h}$ at room temperature and incubated with secondary antibody in block solution at $4{ }^{\circ} \mathrm{C}$ overnight. Embryos were then washed with PBT for at $2 \mathrm{~h}$ at room temperature and stored in $2 \% \mathrm{DABCO}$ (Acros Organics, AC11247-1000).

For 3,3'- diaminobenzidine (DAB) staining, after incubation with primary antibody, samples were incubated in fresh blocking solution with goat anti-rabbit IgG (Covance SMI-5030C, 1:200) at $4{ }^{\circ} \mathrm{C}$ overnight. Embryos were then washed with PBT for $2 \mathrm{~h}$ and incubated with rabbit PAP (Covance SMI-4010 L, 1:200) in block solution at $4{ }^{\circ} \mathrm{C}$ overnight. Embryos were then washed in PBT for $2 \mathrm{~h}$. Staining was performed using Sigma Fast 3,3'- diaminobenzidine tablets (Sigma, D4293).

\section{Imaging}

Embryos were mounted in 70 \% glycerol, $30 \%$ PBS and DIC pictures were taken using an AxioCam MRc5 camera mounted on a Zeiss Axio Imager M1 compound microscope. Fluorescent images were taken on a Zeiss LSM 710 confocal microscope. Images were processed using Adobe Photoshop software (Adobe, Inc) and Image J software (Abramoff et al., 2004). In some cases different focal planes were merged to show labeled cells at different medial lateral positions in the spinal cord.

\section{Cell counts and statistics}

In all cases, cells counts are for both sides of a fivesomite length of the spinal cord adjacent to somites 610. Most values are an average of at least 5 embryos. Exceptions are the skor2 $+\operatorname{Tg}(\operatorname{evxl}: E G F P)$ double-labeling experiments, the skor $2+T g(s l c 17 a 6: E G F P)$ doublelabeling experiments and the pax2a and eng1b in situ hybridization results. In all of these cases 4 embryos were counted. Results were analyzed using the student's $t$-test; Error bars indicate standard deviation.

\section{Results}

\section{Zebrafish V0v cells express evx 1 and evx2}

In mouse, Evx1 is expressed in V0v cells and while double-labeling experiments have not yet been performed, the data suggest that Evx2 is probably coexpressed by these same cells [23, 24, 27, 28]. Previous reports described $e v x 1$ and $e v x 2$ expression in a similar 
region of zebrafish spinal cord [32, 33] but didn't determine whether these genes are co-expressed or the specific cell types that express them.

To address these questions, we performed single and double in situ hybridization experiments and found that zebrafish evx1 and evx2 are co-expressed in an intermediate region of the dorso-ventral axis of the spinal cord (Figs. $1 \mathrm{~g}$ and $2 \mathrm{a} \& \mathrm{i}$ ). We further confirmed that $e v x 1$ and $e v x 2$ are co-expressed in zebrafish spinal cord using an EGFP line, $\operatorname{Tg}(e v x 1: E G F P)^{S U 1}$. We constructed this line using enhancer sequences identified downstream of evx1 (see methods \& Fig. 1d). We confirmed that the stable line recapitulates endogenous evx1 expression (Fig. 1f) and also shows co-expression of EGFP and Evx2 protein (Fig. 1e). Interestingly, at $27 \mathrm{~h}$, all of the cells that express Evx2 also express EGFP (and hence evx1) (Fig. 1e), however, a few cells express EGFP but not Evx2. Similarly, at $24 \mathrm{~h}$, a few cells express evx1 but not evx2 (Fig. 1g). This is consistent with earlier reports that suggest that $e v x 1$ may be expressed in the spinal cord slightly earlier than evx2 [32, 33], although we cannot rule out the possibility that there is a very small subset of evx1-expressing $\mathrm{V} 0 \mathrm{v}$ cells that do not express evx2.

V0 cells develop from the p0 progenitor domain, which expresses $d b x 1[28,63]$. Therefore, to confirm that evx1/2-expressing cells are V0v cells we performed EGFP immunohistochemistry and in situ hybridization for $d b \times 1 a$ and $d b \times 1 b$ in $T g(e v-$ $x$ 1:EGFP) ${ }^{S U 1}$ embryos. We found that zebrafish $e v x$ genes are expressed lateral to cells expressing both of these $d b x 1$ genes, as would be predicted for cells developing from the p0 domain. In addition, $d b \times 1 a$ and $d b \times 1 b$ expression persists in some EGFP-positive cells (Fig. 1h), suggesting that these genes continue to be expressed by V0v cells for a short while after they become post-mitotic.

Zebrafish also have a third evx gene, called eve1, but earlier studies suggested that this gene is not expressed in the spinal cord $[26,32,59,64]$. We confirmed this by

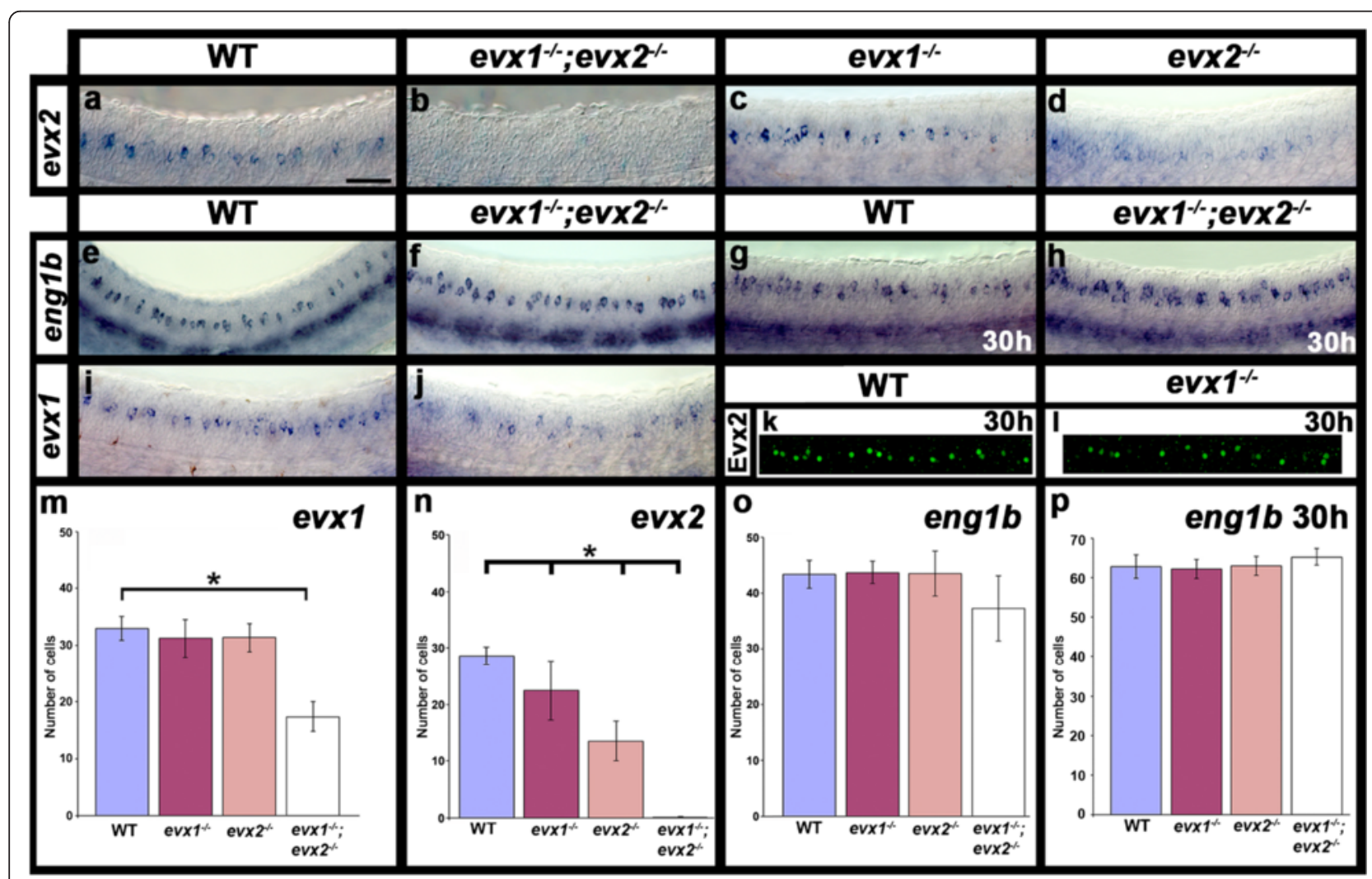

Fig. 2 Expression of evx and eng1b genes in evx1;evx2 double mutant embryos. Lateral views of zebrafish spinal cord at $24 \mathrm{~h}$ (a-f, i \& j) or $30 \mathrm{~h}$ $(\mathbf{g}, \mathbf{h}, \mathbf{k} \& \mathbf{I})$. Anterior left, dorsal up. $\mathbf{a}-\mathbf{j}$ in situ hybridization for each gene indicated. e-h strong ventral expression is in muscle pioneer cells, expression in more individual dorsal cells corresponds to spinal cord V1 cells. $\mathbf{k}$ \& $\mathbf{I}$ immunohistochemistry for Evx2. $\mathbf{m}$-p Average number of cells (y-axis) expressing indicated marker in spinal cord region adjacent to somites 6-10 in WT embryos and evx 1 and evx2 single and double mutants (x-axis) at $24 \mathrm{~h}(\mathbf{m}-\mathbf{o})$ or $30 \mathrm{~h}(\mathbf{p})$. Values are shown as mean $+/$ - standard deviation (values are provided in Table 1). There are no evx2-positive cells in the double mutants (n). In each case at least 5 embryos were counted, except for eng 16 where 4 embryos were counted. Statistically significant differences $(P<0.05)$ from WT values are indicated with brackets and stars. $P$ values for these and other comparisons (e.g differences between single and double mutants) are provided in Table 1. Scale bar: $50 \mu \mathrm{m}(\mathbf{a}-\mathbf{l})$ 
examining eve1 expression at multiple stages of spinal cord development (every two somites from 2-somites 24 h; Fig. 3 and data not shown). In all cases we never saw any spinal cord expression, only expression in the developing tailbud. To check whether eve1 expression is altered in the absence of Evx1 and/or Evx2 we also examined expression in evx1;evx2 double mutants. However, we saw no change in eve1 expression in these double mutants (Fig. 3f). Therefore, this gene is not considered further in this paper. We also confirmed that no additional evx genes exist in zebrafish (Additional file 1: Results and Figure S1).

\section{Zebrafish V0v cells develop into commissural ascending interneurons}

In mouse, V0v cells develop commissural axons that ascend (grow rostrally) for one to four somite segments [23]. We used both the $\operatorname{Tg}(e v x 1 \text { :EGFP })^{S U 1}$ transgenic line and an additional transgenic line that has stronger expression in

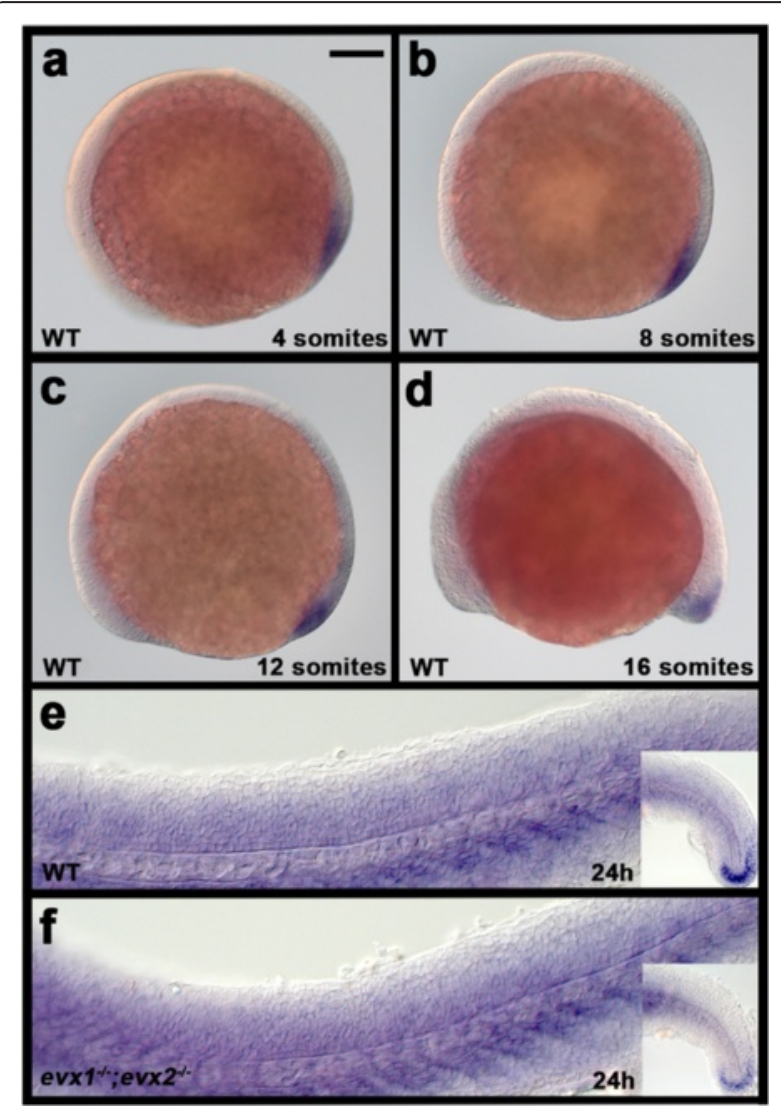

Fig. 3 eve1 is not expressed in zebrafish spinal cord in WT or evx1; evx2 double mutant embryos. Lateral views of in situ hybridization for eve 1 in 4-somite (a), 8-somite (b), 12-somite (c), 16-somite (d) and $24 \mathrm{~h}$ (e \& f) embryos. a-e WT; (f) evx1;evx2 double mutant. Anterior is left and dorsal up. Expression is seen in the tail bud region but not the spinal cord. Embryos in (e \& f) were over-stained to check that there was no weak expression in the spinal cord. The only specific staining seen was at the end of the tail (inset in right bottom corner). The rest is background staining from over-staining. Scale bar: $100 \mu \mathrm{m}$
V0v cell axons, $\operatorname{Tg}(\text { evx } 1: E G F P)^{S U 2}$ (see methods), to examine the morphology of zebrafish V0v cells. We found that by 27-30 h, almost all of the cells have extended their axons ventrally and have at least started to cross the midline to the other side of the spinal cord (Fig $4 \mathrm{a} \& \mathrm{~b}$ ). By $48 \mathrm{~h}$, most of the cells have reached the other side of the spinal cord and have turned towards the head, giving them a clear commissural ascending, or CoSA $[65,66]$, morphology (Fig. 4c \& f).

\section{V0v cells are glutamatergic}

In wild-type mouse spinal cords, V0 cells develop into both inhibitory (glycinergic or GABAergic) and excitatory (glutamatergic) interneurons [27, 28, 36], but when we started this project the neurotransmitter phenotype of $\mathrm{VOv}$ cells had not been established. Zebrafish have both excitatory and inhibitory CoSA interneurons $[61,62]$, so we could not infer the neurotransmitter properties of V0v cells from their morphology alone. Therefore, we performed double-labeling experiments to establish the neurotransmitter fates of these cells. Double immunohistochemistry for Evx2 and EGFP in the $\operatorname{Tg}$ (slc17a6:EGFP) line which labels glutamatergic interneurons in the zebrafish spinal cord [37,67] revealed that all of the Evx2-positive cells are glutamatergic (Fig. 5a). As expected, not all of the glutamatergic cells express Evx2, since there are several excitatory cell types in the zebrafish spinal cord and only V0v cells express Evx2. In addition, in situ hybridization for slc17a6 (vglut) genes, markers of glutamatergic cells (see methods for more details) combined with EGFP immunohistochemistry in $\operatorname{Tg}(e v x 1 \text { :EGFP })^{S U 1}$ embryos also confirmed that evx1-expressing cells are glutamatergic (Fig. 5b). We also examined if evxl is coexpressed with markers of any other spinal cord neurotransmitter fates. Using double in situ hybridization we found no co-expression between $e v x 1$ and markers for glycinergic or GABAergic markers (data not shown). We also observed no double-labeled cells when we performed EGFP immunohistochemistry and in situ hybridization for slc32a1 (formerly called viaat) which labels all inhibitory neurons $[8,68,69]$ in $\operatorname{Tg}(e v x 1: E G F P)^{S U 2}$ embryos (Fig. 5c).

Consistent with these analyses, when we FAC-sorted and expression profiled EGFP-labeled V0v cells using the $\operatorname{Tg}(e v x 1: E G F P)^{S U 1}$ line we found that these cells express markers of glutamatergic fates (slc17a6a (formerly called vglut2.2) and slc17a6b (formerly called vglut2.1)) and do not express either glycinergic markers (slc6a9 (formerly called glyt1) or slc6a5 (formerly called glyt2)) or GABAergic markers (gad1b or gad2) (Fig. 5d \& e).

\section{Zebrafish V0v cells do not express Pax2}

As discussed above, Pax2 is an important regulator of inhibitory spinal cord fates [14-21]. Given that we had shown that V0v cells are excitatory (glutamatergic) and we had not observed any inhibitory V0v cells 


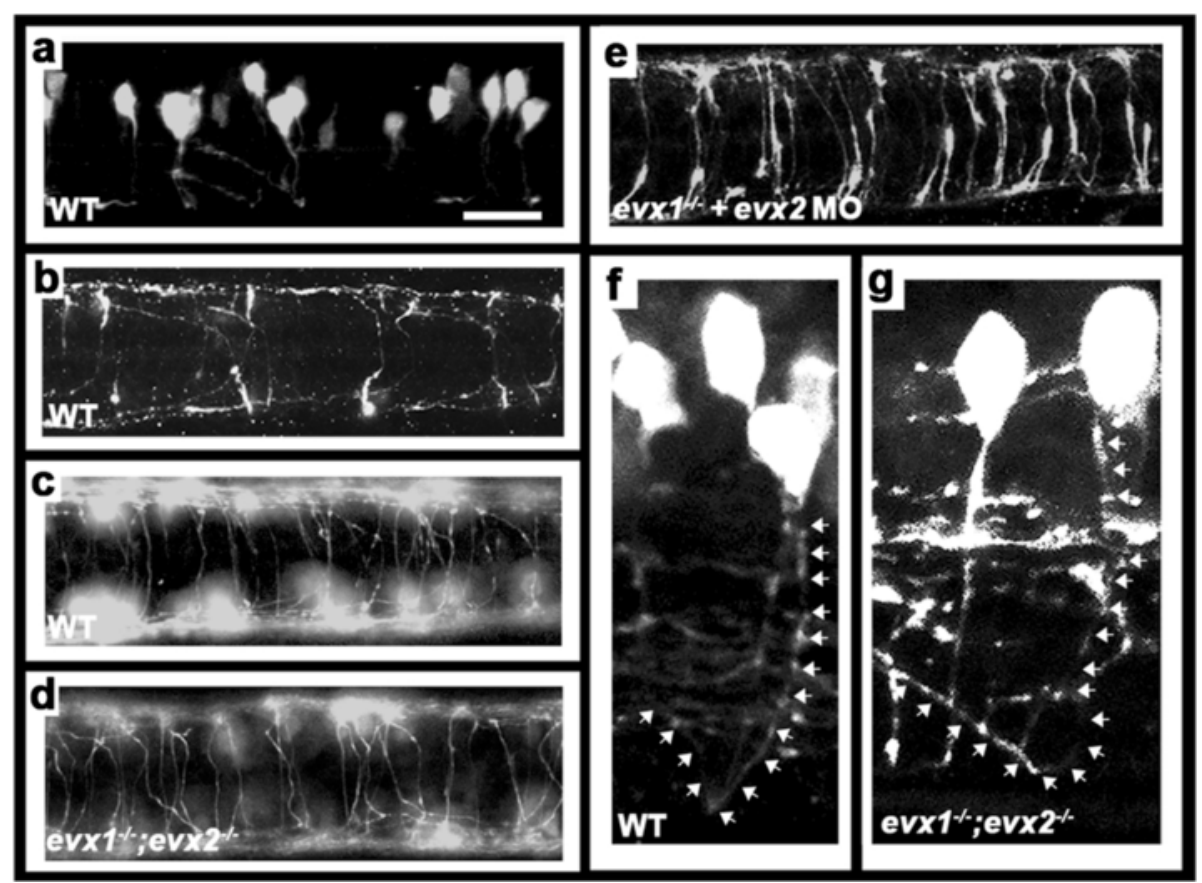

Fig. 4 VOv cells develop into CoSA interneurons. Immunohistochemistry for EGFP in Tg(evx1:EGFP) ${ }^{\text {SU1 }}$ (a, b \& e) or Tg(evx1:EGFP) ${ }^{\text {SU2 }}$ (c, d, $\mathbf{f}$ \& $\left.\mathbf{g}\right)$ embryos:a, $\mathbf{f} \& \mathbf{g}$ lateral views with dorsal up and anterior left of spinal cord at $27 \mathrm{~h}$ (a) or $48 \mathrm{~h}(\mathbf{f} \& \mathbf{g})$. $\mathbf{b}$-e dorsal views with anterior left of zebrafish spinal cord at $30 \mathrm{~h}$ (b) or $48 \mathrm{~h}$ (c-e). a-c \& f WT, (d \& g) evx1;evx2 double mutant, (e) evx1 mutant injected with evx2 morpholino. b \& c show increasing number of commissural axons crossing the spinal cord as development proceeds. $\mathbf{d} \&$ e demonstrate that V0v axons are still clearly commissural in the absence of Evx 1 and Evx2. $\mathbf{f} \& \mathbf{g}$ show magnified views of commissural ascending Vov axons. White arrows (drawn slightly to the right of the axon so that EGFP expression is still visible) indicate ascending axon trajectories. Scale bar: $50 \mu \mathrm{m}(\mathbf{a}-\mathbf{e})$ and $15 \mu \mathrm{m}(\mathbf{f} \& \mathbf{g})$

(Figs 5a-e), we would not predict that V0v cells would express Pax2. However, the literature contains contradictory evidence as to whether $e v x 1$ and $e v x 2$ are co-expressed with pax 2 in the spinal cord [35, 58, 70, 71]. To resolve this issue, we performed double-labeling experiments for $e v x 1$ and pax2 using several complementary approaches. These included in situ hybridization for $e v x 1$ and immunohistochemistry for Pax2, EGFP immunohistochemistry and in situ hybridization for $\operatorname{pax} 2 a, \operatorname{pax} 2 b$ and pax8 (three highly-related pax genes that are co-expressed in zebrafish spinal cord cells [14]) in $\operatorname{Tg}(e v x 1: E G F P)^{S U 2}$ embryos and double immunohistochemistry for EGFP and Pax2 in $\operatorname{Tg}(\text { evx } 1: E G F P)^{S U 2}$ embryos. In all cases, we observed no double-labeled cells, suggesting that $e v x 1$ and $e v x 2$ are not co-expressed with pax2/ pax8 genes in zebrafish spinal cord (Fig. $6 \mathrm{~g}$ and data not shown). These analyses complement those of Satou and colleagues [36] who recently reported that inhibitory V0 cells, which presumably correspond to $\mathrm{V} 0_{\mathrm{D}}$ cells, express Pax2, but Evx2-expressing cells do not. Taken together, these data strongly suggest that V0v cells do not express pax2 genes.

evx $2^{\text {sa140 }}$ is a null allele

To identify the functions of $e v x 1$ and $e v x 2$ in zebrafish V0v cells we used $e v x 1$ and $e v x 2$ mutants (see Methods).
Our previous analyses suggest that $e v x 1^{i 232}$ is a null allele [39]. The $e v x 2^{\text {sal40 }}$ mutation introduces a premature stop codon just before the homeodomain, suggesting that if a truncated protein is synthesized it will have no DNA binding activity. However, it is possible that a truncated protein might retain some function in the embryo. To determine if any Evx2 protein is made in mutant embryos, we used an Evx2 antibody that was made against the first 168 amino acids of zebrafish Evx2 (which corresponds exactly to the region upstream of the premature stop codon in the evx $2^{\text {sa140 }}$ mutant allele). We found that all of the WT (21/51) and heterozygous embryos (20/51) had Evx2 antibody staining but all of the homozygous mutants (10/51) did not (Fig. 1c). This strongly suggests that the evx2 mutant allele does not produce any protein and is a null allele.

\section{evx $2^{\text {sa140 }}$ homozygous mutants are not viable}

Unlike the zebrafish evx1 mutant, which is homozygous viable [39], we never identified an adult evx2 homozygous mutant ( $\mathrm{n}=262$ fish from incrosses of heterozygous evx2 fish, $\mathrm{P}<0.0001$ using chi-squared test). However, we did obtain fish homozygous for evxl and heterozygous for evx2 (17 fish identified from a total of 191 fish from incrosses of heterozygous double mutants; $\mathrm{P}=0.13$ using 


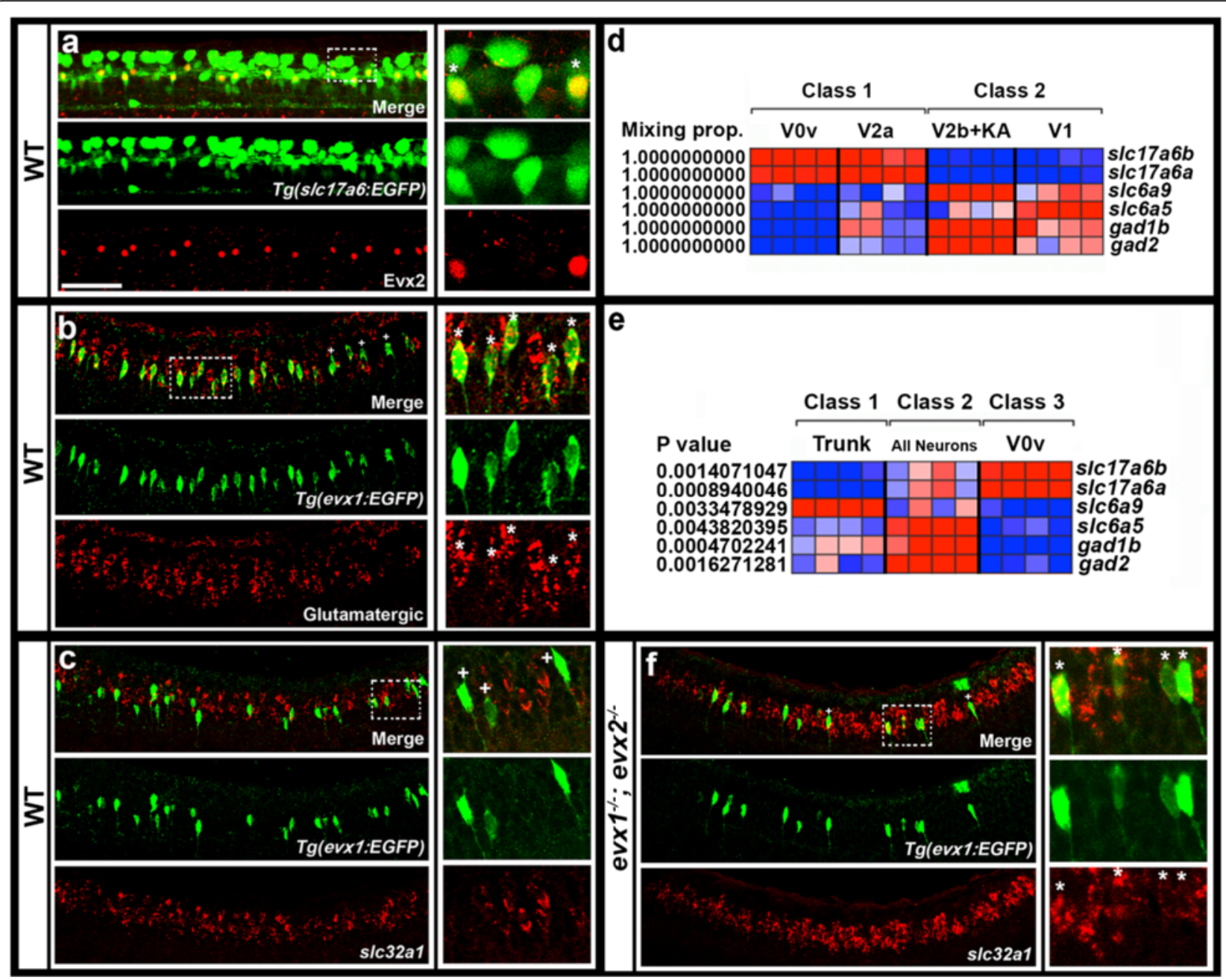

Fig. 5 Neurotransmitter phenotypes of VOv cells. Lateral views of spinal cord at $27 \mathrm{~h}(\mathbf{a} \& \mathbf{b})$ or $30 \mathrm{~h}$ (c \& $\mathbf{f}$ ). All panels contain merged and single channel views. Smaller images on RHS are single confocal planes of white box regions. Double and single-labeled EGFP-positive cells are indicated in single confocal planes with stars and crosses respectively. a EGFP (green) and Evx2 (red) expression in Tg(s/c17a6:EGFP) embryo. All Evx2-positive cells co-express EGFP. b EGFP (green) and glutamatergic marker (slc17a6b \& s/c17a6a; red) expression in Tg(evx1:EGFP) ${ }^{\text {SU1 }}$ embryo. Occasional single-positive cells are indicated with crosses. These may be expressing glutamatergic markers at levels too low to detect (s/c17a6 probes are weak in doublelabeling experiments). Remaining cells are double-labeled. c \& f EGFP (green) and s/c32a1 (red) expression in Tg(evx1:EGFP) ${ }^{\mathrm{SU2}}$ WT (c) and evx1;evx2 double mutant (f) embryos. No VOv cells are inhibitory in WT embryos, but most VOv cells are inhibitory in double mutants (occasional single-labeled cells are indicated with a cross in F). (d \& e) Relative expression profiles of genes (names on right) indicative of neurotransmitter fates at $27 \mathrm{~h}$. Columns represent individual microarray experiments. Rows indicate relative expression levels as normalized data transformed to mean of zero and standard deviation of +1 (highly-expressed, red) to -1 (weakly/not expressed, blue) sigma units. For details of how cells were isolated see methods. $\mathbf{d}$ Two-class eBayes comparison of excitatory (class 1) versus inhibitory (class 2) cells. Mixing proportion measures posterior probability, or likelihood that genes are differentially expressed ( 1 = highest probability of differential expression). e Three-class ANOVA comparison of VOv cells (class 3 ) versus trunk cells (class 1) and all post-mitotic neurons (class 2). P values test hypothesis that there is no differential expression between the 3 classes. VOv cells express glutamatergic markers s/c17a6a and s/c17a6b and do not express glycinergic or GABAergic markers in both comparisons. Scale bar: $50 \mu \mathrm{m}$ (a-c \& $\mathbf{f}$ )

chi-squared test). Our analyses of incrosses from identified evx2 heterozygous fish suggest that evx2 mutant embryos have no obvious morphological defects for the first few days of development but that most of them die by larval stages (see Additional file 1: Results).

V0v cells still form in evx1;evx2 double mutants In mouse Evx1 mutants, expression of Evx2 is lost and there is an increase in the number of cells expressing the V1 marker En1. In addition, many of the cells that would normally have expressed Evx1 develop axon trajectories similar to V1 cells. Most strikingly their axons change from being commissural to ipsilateral [23]. This suggests that in the absence of Evx1, most mouse V0v cells transfate to V1 cells.

In contrast, we found that in zebrafish evx1 mutants there is only a small reduction in the number of cells expressing evx2 RNA and Evx2 protein in the spinal cord 


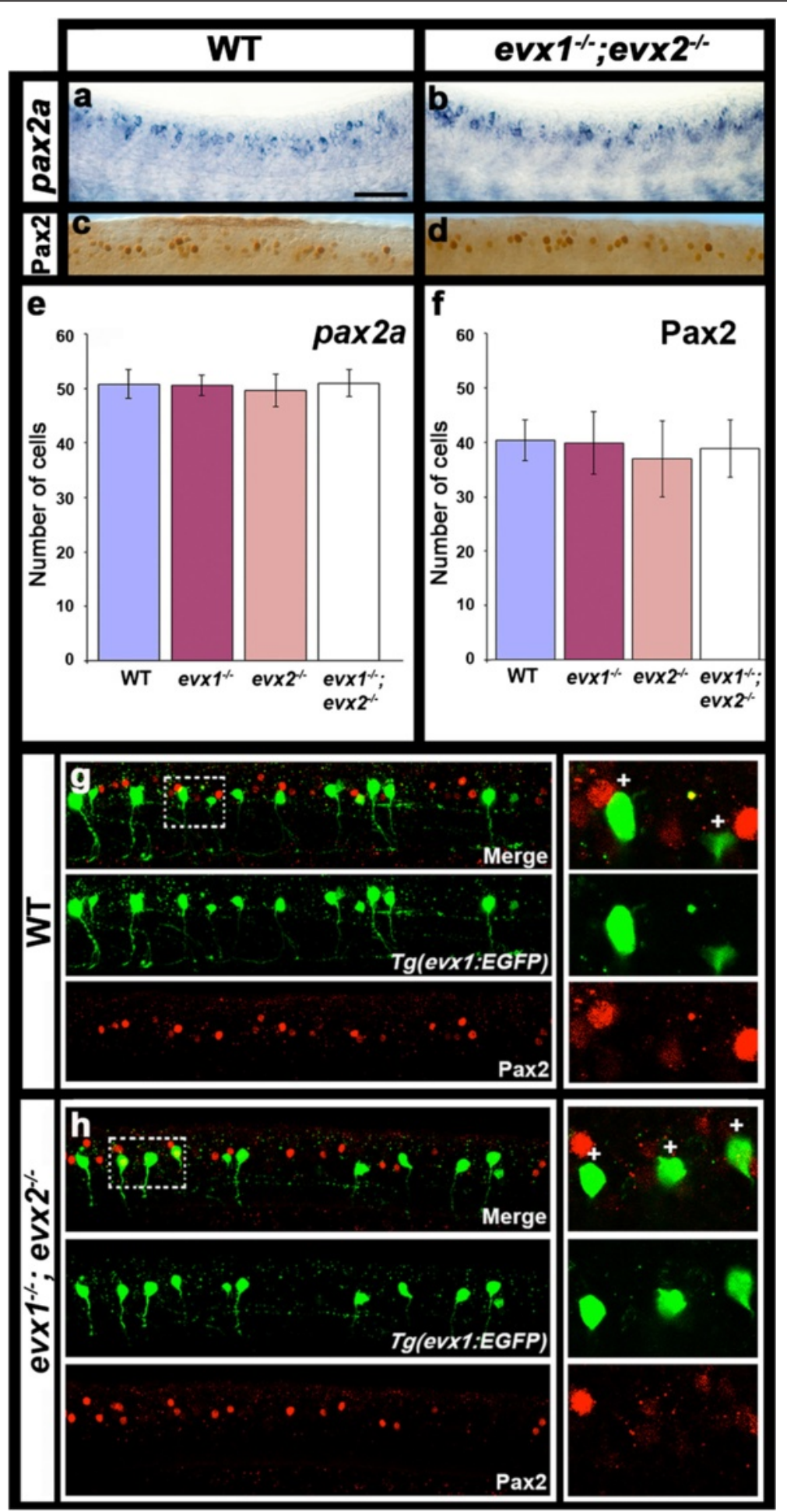

Fig. 6 VOv cells do not express Pax2 in WT or evx1; evx2 double mutant embryos. Lateral views of zebrafish spinal cord at $24 \mathrm{~h}$ (a-d) or $30 \mathrm{~h}$ (g \& h). Anterior left, dorsal up. a \& b in situ hybridization for pax2a. c \& d immunohistochemistry for Pax2. The Pax2 antibody recognizes both Pax2a and Pax2b. e \& $\mathbf{f}$ Average number of cells (y-axis) expressing these markers (indicated in each case) in spinal cord region adjacent to somites 6-10 in WT embryos and evx1 and evx2 single and double mutants (x-axis). Values are shown as mean $+/$ - standard deviation (values are provided in Table 1). In each case at least 5 embryos were counted, except for pax2a where 4 embryos were counted. P values for all comparisons are provided in Table 1. $\mathbf{g}$ \& h EGFP (green) and Pax2 (red) expression in Tg(evx1:EGFP) ${ }^{\mathrm{SU} 2}$ WT (g) and evx1;evx2 double mutant (h) embryos. No V0v cells express Pax2 in either case. Panels on RHS are magnified single-confocal-plane views of white dotted rectangle regions in panels $\mathrm{G}$ and $\mathrm{H}$ respectively. White crosses indicate single-positive GFP cells. Scale bar: $50 \mu \mathrm{m}(\mathbf{a}-\mathbf{d}) \& 40 \mu \mathrm{m}(\mathbf{g}-\mathbf{h})$ 
(approximately a $22 \%$ reduction for $e v x 2$ at $24 \mathrm{~h}$ and a $32 \%$ reduction for Evx2 at 30 h; Fig. 2c, 1 \& n; Table 1), although expression of $e v x 2$ RNA is lost completely in evx1;evx2 double mutants (Fig. $2 \mathrm{~b} \& \mathrm{n}$; Table 1). In contrast, there is no difference in evx1 expression in $e v x 1$ or evx2 single mutants when compared to WT siblings, although approximately $47 \%$ of V0v cells lose expression of evx1 in double mutants (Fig. 2j \& m; Table 1). Consistent with the down-regulation of evx1 in double mutants, we observe a $30 \%$ reduction in the number of EGFP-labeled V0v cells in double mutant $\mathrm{Tg}(\mathrm{ev}$ $x 1: E G F P)^{S U 2}$ embryos $(22.9+/-3.4$ cells in WTs; $16.0+/-$ 5.5 cells in double mutants). However, strikingly, most V0v cells are still labeled with EGFP and these cells have a normal commissural ascending CoSA morphology (Fig. 4d \& g). Unfortunately, we were never able to identify double mutant embryos that carried the $\mathrm{Tg}(\mathrm{ev}$ $x 1$ :EGFP) ${ }^{S U 1}$ transgene, suggesting that this transgene probably integrated in the vicinity of the WT evx2 allele (see methods). However, consistent with our results using the $\operatorname{Tg}(\operatorname{evx} 1: E G F P)^{S U 2}$ transgenic line, when we injected evx2 ATG morpholinos, at a concentration that eliminates Evx2 protein, into evx1 single mutant $\mathrm{Tg}(\mathrm{ev}$ $x 1: E G F P)^{S U 1}$ embryos, we also observed EGFP-labeled V0v cells with normal CoSA axon trajectories (Fig. 4e).

Consistent with this persistence of V0v cells, we also found no change in the number of eng1b-expressing spinal cord cells in evx1 and evx2 single or double mutants compared to WT embryos at $24 \mathrm{~h}$. If anything, we observed a slight reduction in the number of eng $1 b$ cells in the double mutants, although this was not statistically significant (Fig. $2 \mathrm{f} \& \mathrm{o}$, Table 1). To further confirm that V0v cells were not adopting a V1 fate and turning on eng1b expression, we repeated this experiment at $30 \mathrm{~h}$. We still found no change in eng1b expression in either single or double mutants when compared to WT embryos (Fig. 2h \& p, Table 1).

Taken together, these results suggest that V0v cells are not transfating into V1 cells in zebrafish, even in the absence of both Evx1 and Evx2. Instead at least most of these cells are maintaining their V0v identities. In addition, these data suggest that Evx1 and Evx2 act partially redundantly to maintain each other's expression, although only $e v x 2$ expression requires Evx1/Evx2 activity as more than half of $\mathrm{VOv}$ cells still express evx1 in evx1;evx2 double mutants.

\section{Evx1 and Evx2 are required for skor2 expression in V0v cells}

To identify additional transcription factors that might be required for specification of $\mathrm{V} 0 \mathrm{v}$ functional characteristics, we expression-profiled FAC-sorted V0v cells (see Methods; [50]). From these analyses, we identified skor 2 as a transcription factor gene potentially expressed by V0v neurons.
Our subsequent in situ hybridization experiments demonstrated that skor2 has two clear domains of spinal cord expression, a ventral domain and a more dorsal domain (Fig. 7c). Double labeling experiments show that in the ventral domain, at least most of the skor2-expressing cells are V0v cells (Fig. 7a). On average, 97 \% of ventral skor2expressing cells co-express EGFP in $\operatorname{Tg}(\text { evx1:EGFP })^{\text {SU1 }}$ embryos (73/75 cells counted in 4 embryos). Given this high number of double positive cells and the fact that there is usually a delay in EGFP expression it is possible that all of the ventral skor2-expressing cells are V0v cells. Interestingly, double labeling experiments with skor2 and $\mathrm{Tg}$ (slc17a6:EGFP) demonstrated that both the ventral and dorsal skor2-expressing cells are excitatory cells (Fig. 7b), suggesting that Skor2 may play in role in specifying excitatory fates. As skor 2 is expressed by V0v cells, we tested whether it is regulated by Evx1 and Evx2. We found that the number of cells expressing skor 2 in the ventral spinal cord is reduced in evx1 and evx2 single mutants compared to WT embryos. More strikingly, ventral skor2 expression is completely abolished in double evx1;evx2 mutants (Figs 7d \& e; Table 1), demonstrating that Evx1 and Evx2 are required, partially redundantly for skor 2 expression in V0v cells. In contrast, there was no change in the dorsal expression of skor2 (Fig. 7d \& e; Table 1).

\section{Evx1 and Evx2 are required to specify the glutamatergic fates of Vov cells}

Given that V0v cells still form in the absence of Evx1 and Evx2 function and their axon trajectories appear to be unaffected, but expression of a novel excitatory cell marker skor2 is lost, we decided to test if V0v cell neurotransmitter phenotypes were changed. When we examined the expression of slc17a6 (vglut) genes in embryos from a cross of evx1;evx2 heterozygous parents, we saw a significant reduction of glutamatergic cells in both of the single mutants when compared to WT embryos and an even more severe reduction in double mutants (Fig. 8a-d \& o; Table 1). These data indicate that Evx1 and Evx2 act partially redundantly to specify the glutamatergic phenotype of V0v cells. Strikingly, the number of glutamatergic cells lost in the double mutant (approximately 28 cells in the spinal cord region adjacent to somites 6-10) is equivalent to the number of $\mathrm{VOV}$ cells in that region of the spinal cord (approximately 29 cells express evx2 and 33 cells express $e v x 1$ in this region of the WT spinal cord at this stage; see Table 1), suggesting that probably all of the V0v cells have lost their glutamatergic phenotype.

\section{V0v cells become inhibitory in evx1;evx double mutant embryos}

Given that V0v cells lose their excitatory phenotype in evx1;evx2 double mutants, we asked whether they are acquiring an inhibitory neurotransmitter fate instead. 
Table 1 Number of cells expressing particular genes and proteins in WT and mutant embryos

\begin{tabular}{|c|c|c|c|c|c|c|c|c|c|c|}
\hline Marker & Stage & WT & evx1 mutants & $P^{a}$ & evx2 mutants & $\mathrm{P}^{b}$ & Double mutants & $P^{c}$ & $\mathrm{P}^{\mathrm{d}}$ & $\mathrm{pe}^{\mathrm{e}}$ \\
\hline evx1 & $24 \mathrm{~h}$ & $33.0+/-2.0$ & $31.0+/-3.3$ & 0.32 & $31.3+/-2.5$ & 0.56 & $17.4+/-2.6$ & $<0.01$ & $<0.01$ & $<0.01$ \\
\hline evx2 & $24 \mathrm{~h}$ & $28.6+/-1.6$ & $22.4+/-50$ & 0.02 & $13.5+/-3.5$ & $<0.01$ & 0.00 & $<0.01$ & $<0.01$ & $<0.01$ \\
\hline Evx2 & $30 \mathrm{~h}$ & $33.6+/-5.2$ & $23.0+/-4.6$ & 0.01 & N.D & & N.D & & & \\
\hline eng1b & $24 \mathrm{~h}$ & $43.3+/-2.5$ & $43.6+/-2.0$ & 0.80 & $43.5+/-4.0$ & 0.94 & $37.2+/-6.0$ & 0.08 & 0.07 & 0.10 \\
\hline eng1b & $30 \mathrm{~h}$ & $63.0+/-3.0$ & $62.0+/-2.5$ & 0.63 & $63.0+/-2.5$ & 0.92 & $65.2+/-2.0$ & 0.15 & 0.06 & 0.15 \\
\hline slc17a6 (vlgut) & $24 \mathrm{~h}$ & $89.0+/-10.0$ & $71.3+/-4.3$ & $<0.01$ & $74.6+/-8.0$ & 0.01 & $60.8+/-10$ & $<0.01$ & 0.08 & 0.04 \\
\hline slc32al (viaat) & $24 \mathrm{~h}$ & $157.4+/-8.0$ & $157.3+/-10.0$ & 0.75 & $158.2+/-4.0$ & 0.86 & $178.6+/-7.0$ & $<0.01$ & $<0.01$ & $<0.01$ \\
\hline gads (GABAergic) & $24 \mathrm{~h}$ & $50.0+/-3.0$ & $49.0+/-4.0$ & 0.59 & $51.6+/-2.6$ & 0.31 & $50.6+/-2.2$ & 0.66 & 0.38 & 0.5 \\
\hline slc6a5 (glyt2a/glyt2b) & $24 \mathrm{~h}$ & $81.0+/-3.3$ & $93.0+/-9.0$ & $<0.01$ & $87.6+/-4.5$ & 0.02 & $109+/-10.0$ & $<0.01$ & 0.02 & $<0.01$ \\
\hline pax2a & $24 \mathrm{~h}$ & $50.6+/-2.6$ & $50.4+/-2.0$ & 0.86 & $49.5+/-30$ & 0.55 & $50.8+/-2.5$ & 0.91 & 0.75 & 0.49 \\
\hline $\operatorname{Pax2}$ & $24 \mathrm{~h}$ & $40.3+/-3.8$ & $39.8+/-5.7$ & 0.83 & $36.9+/-6.9$ & 0.23 & $38.8+/-5.3$ & 0.41 & 0.68 & 0.52 \\
\hline skor2 & $30 \mathrm{~h}$ & $24.3+/-3.0$ & $15.0+/-5.0$ & $<0.01$ & $19.8+/-4.0$ & 0.07 & 0.0 & $<0.01$ & $<0.01$ & $<0.01$ \\
\hline skor2 Total cell counts & $30 \mathrm{~h}$ & $46.7+/-3.7$ & $38.7+/-3.3$ & $<0.01$ & $42.4+/-1.7$ & 0.04 & $22.6+/-1.7$ & $<0.01$ & $<0.01$ & $<0.01$ \\
\hline
\end{tabular}

Numbers of cells expressing particular markers (first column on left) in spinal cord region adjacent to somites 6-10 and P values of comparisons between embryos with different genotypes. Values are shown as the mean from at least 5 different embryos $+/$ - standard deviation, except for pax $2 a$ and eng $1 b$ where 4 embryos were counted. $P$ values are from student's $t$-tests. Statistically significant $(P<0.05)$ values are indicated in bold. $P^{a}$ compares evx 1 single mutants with WT embryos, $\mathrm{P}^{\mathrm{b}}$ compares evx2 single mutants with WT embryos, $\mathrm{P}^{\mathrm{c}}, \mathrm{P}^{\mathrm{d}}$ and $\mathrm{P}^{\mathrm{e}}$ are for comparisons between double mutant embryos and WT ( $\mathrm{P}^{\mathrm{C}}$ ), evx1 single mutant $\left(P^{d}\right)$ and evx2 single mutant $\left(P^{\mathrm{e}}\right)$ embryos respectively. Mean cell count values are provided to one decimal place and $P$ values to two decimal places. For skor2, two sets of values are provided: just the ventral domain of expression and both the ventral and dorsal domains of expression (total cell counts)

When we examined expression of slc32a1, which is expressed by all inhibitory neurons $[8,68,69]$, there was no significant difference in the number of cells expressing this gene between either of the single mutants and WT embryos (Fig. 8e, g, h \& p, Table 1). However, interestingly, there was a significant increase (approximately 21 cells) in the number of slc32a1-expressing cells in evx1;evx2 double mutants (Fig. 8f \& p, Table 1). This suggests that Evx1 and Evx2 act redundantly to repress the inhibitory fate in VOv cells.

To further confirm that V0v cells are switching to an inhibitory fate, we performed in situ hybridization for slc32a1 plus immunohistochemistry for EGFP in embryos from a cross of double heterozygous parents that carry the $\operatorname{Tg}(\operatorname{evx1:EGFP})^{S U 2}$ transgene. In WT embryos we see no co-expression of slc32al and EGFP (Fig. 5c). However, in double mutant embryos most V0v cells express slc32a1 (77\% of EGFP-positive V0v cells (30/39 cells counted in 2 embryos); Fig. 5f).

To determine whether V0v cells are becoming GABAergic and/or glycinergic we examined expression of markers of these two fates. We see no significant difference in the number of cells expressing GABAergic markers in single or double mutant embryos (Fig. $8 \mathrm{~m}$, $\mathrm{n} \& \mathrm{r}$ ). In contrast, there is an increase in the number of cells expressing glycinergic markers. Interestingly, and in contrast to the slc32a1 (viaat) result, we see a slight increase in both single mutants as well as a more pronounced increase in double mutants (Fig. 8i-1 \& q, Table 1). The increase in double mutants (approximately 28 cells) suggests that all V0v cells are becoming glycinergic.

\section{V0v cells become glycinergic through a novel Pax2-} independent mechanism

All of the transcription factors that have been identified so far as specifying inhibitory spinal fates act through Pax2 [14-21]. In addition, Tlx1 and Tlx3, the only other transcription factors that have been identified as specifying excitatory spinal cord fates [4, 5, 8], work at least in part by down-regulating Pax2 [4]. Therefore, we decided to test if V0v cells turn on Pax2 expression in evx1;evx2 double mutants. However, when we analyzed pax $2 a$ expression there was no significant difference in the number of cells expressing this gene in either the single or double mutants compared to WT embryos (Fig. 6b \& e; Table 1). To further confirm this result, we performed immunohistochemistry using a Pax2 antibody that recognizes Pax2a and Pax2b [55]. Again, we saw no significant change in the number of cells expressing Pax2 protein in single or double mutants (Fig. 6d \& f; Table 1). Finally, we also performed double-labeling experiments for Pax2 and EGFP in WT and mutant embryos that carried the $\operatorname{Tg}(\operatorname{evx1:EGFP})^{S U 2}$ transgene, using either the Pax2 antibody or in situ hybridization with a mix of $p a x 2 a, p a x 2 b$ and pax 8 probes. In each case we examined at least two WT embryos and two double homozygous mutants and we did not observe any doublelabeled cells (Fig. 6h and data not shown).

Taken together these results show that V0v cells are becoming glycinergic through a Pax2-independent mechanism. This is the first time that a Pax2independent mechanism of glycinergic specification has been identified in spinal cord neurons. 


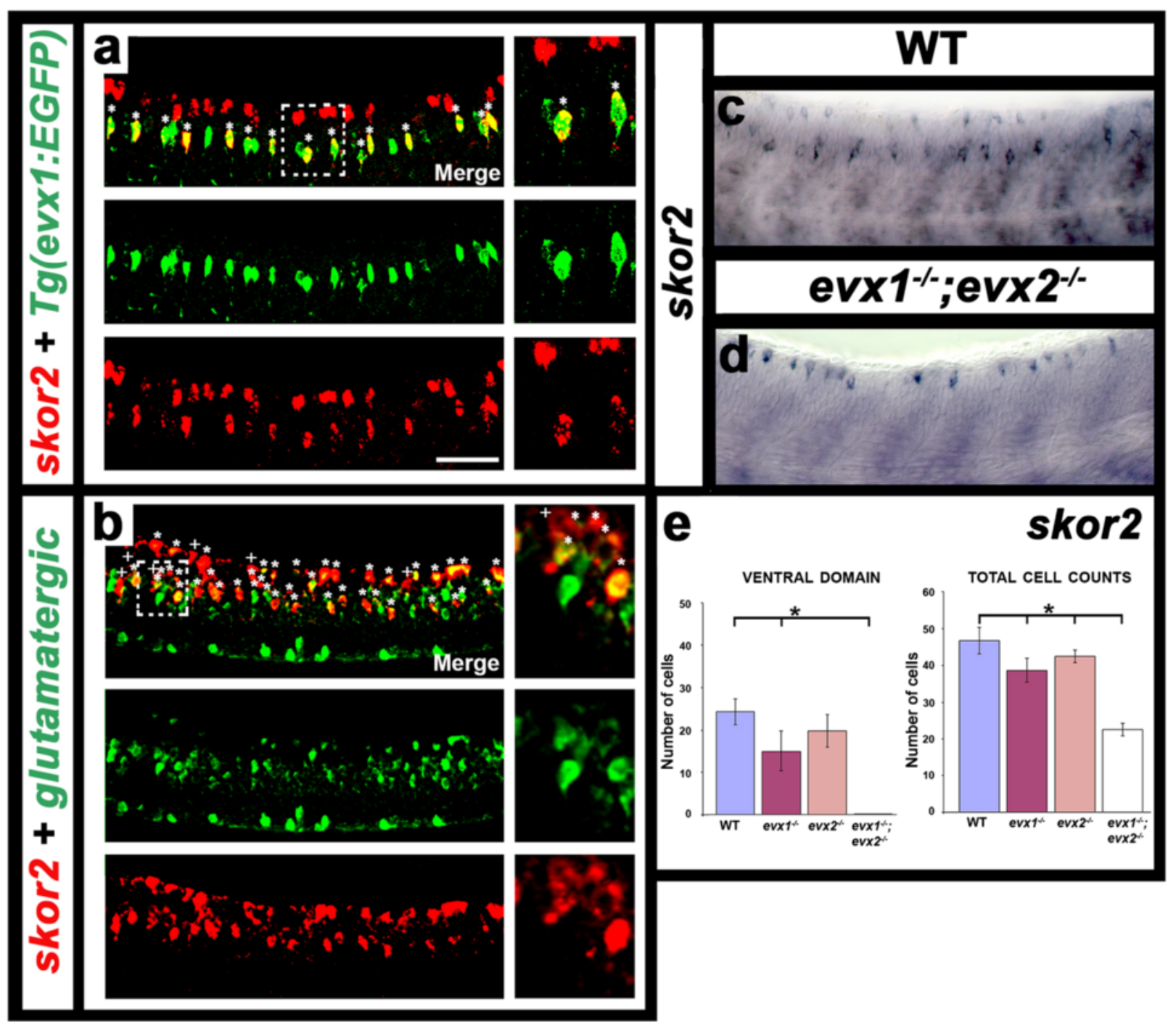

Fig. 7 skor2 is expressed by Vov cells and this expression is lost in evx1;evx2 double mutants. Lateral views of zebrafish spinal cord at $27 \mathrm{~h} \mathrm{(a)} \mathrm{and} 30 \mathrm{~h} \mathrm{(b-d).} \mathrm{Anterior} \mathrm{left,} \mathrm{dorsal} \mathrm{top.} \mathrm{a} \mathrm{\&} \mathrm{b} \mathrm{Merged} \mathrm{images} \mathrm{on} \mathrm{top} \mathrm{followed} \mathrm{by} \mathrm{single-channel} \mathrm{views.} \mathrm{Panels} \mathrm{on} \mathrm{RHS} \mathrm{are}$ single confocal planes of white dashed-box regions. Stars indicate double-positive cells. a Expression of skor2 (red) and EGFP (green) in Tg(evx1:EGFP) $)^{\text {SU1 }}$ embryo. In this example, all ventral skor2-expressing cells co-express EGFP. On average, $97 \%$ of ventral skor2-expressing cells co-express EGFP (73/75 cells counted in 4 embryos). In contrast, about $57.5 \%$ of Vov cells co-express skor2 (73/127 cells counted in 4 embryos). b Expression of skor2 (red) and EGFP (green) in Tg(s/c17a6:EGFP) embryo that labels glutamatergic cells. Crosses indicate cells that are only clearly positive for skor2. On average $93.5 \%$ of skor2-expressing cells co-express EGFP (201/215 cells counted in 4 embryos). As there is usually a delay in EGFP expression it is possible that all ventral skor2-expressing cells are excitatory Vov cells, as the small number of ventral skor2-positive EGFP-negative cells may be just starting to express EGFP. c \& $\mathbf{d}$ Expression of skor2 (blue) in both WT (c) and evx1;evx2 double mutant (d) embryos. The ventral row of skor2 expression is lost in double mutants. e Average number of cells (y-axis) expressing skor2 in spinal cord region adjacent to somites 6-10 in WT embryos and evx1 and evx2 single and double mutants (x-axis) at $30 \mathrm{~h}$. Results are shown for the ventral (VOv) domain of skor2 expression and for the whole skor2 expression domain (total cell counts). Values are mean +/- standard deviation (also see Table 1). In each case at least 5 embryos were counted. Statistically significant differences $(P<0.05)$ from WT values are indicated with stars. $P$ values for all comparisons are provided in Table 1. Scale bar: $50 \mu m$ $(\mathbf{a} \& \mathbf{b}) ; 40 \mu \mathrm{m}(\mathbf{c} \& \mathbf{d})$

\section{Discussion}

$E v x$ genes are found in a wide range of animals ranging from corals to humans [72]. They encode transcription factors that contain both DNA-binding homeobox and C-terminal repressor domains [73-77]. Amniotes have two $E v x$ genes (Evx1 and $E v x 2)$ and teleosts, including zebrafish, have three (evx1, evx2 and eve1), although only $e v x 1$ and $e v x 2$ are expressed in the spinal cord ([26, 32, 33, 59, 64]; this paper Figs. 2, 3, Additional file 1: Results and Figure S1). Interestingly, the genomic 


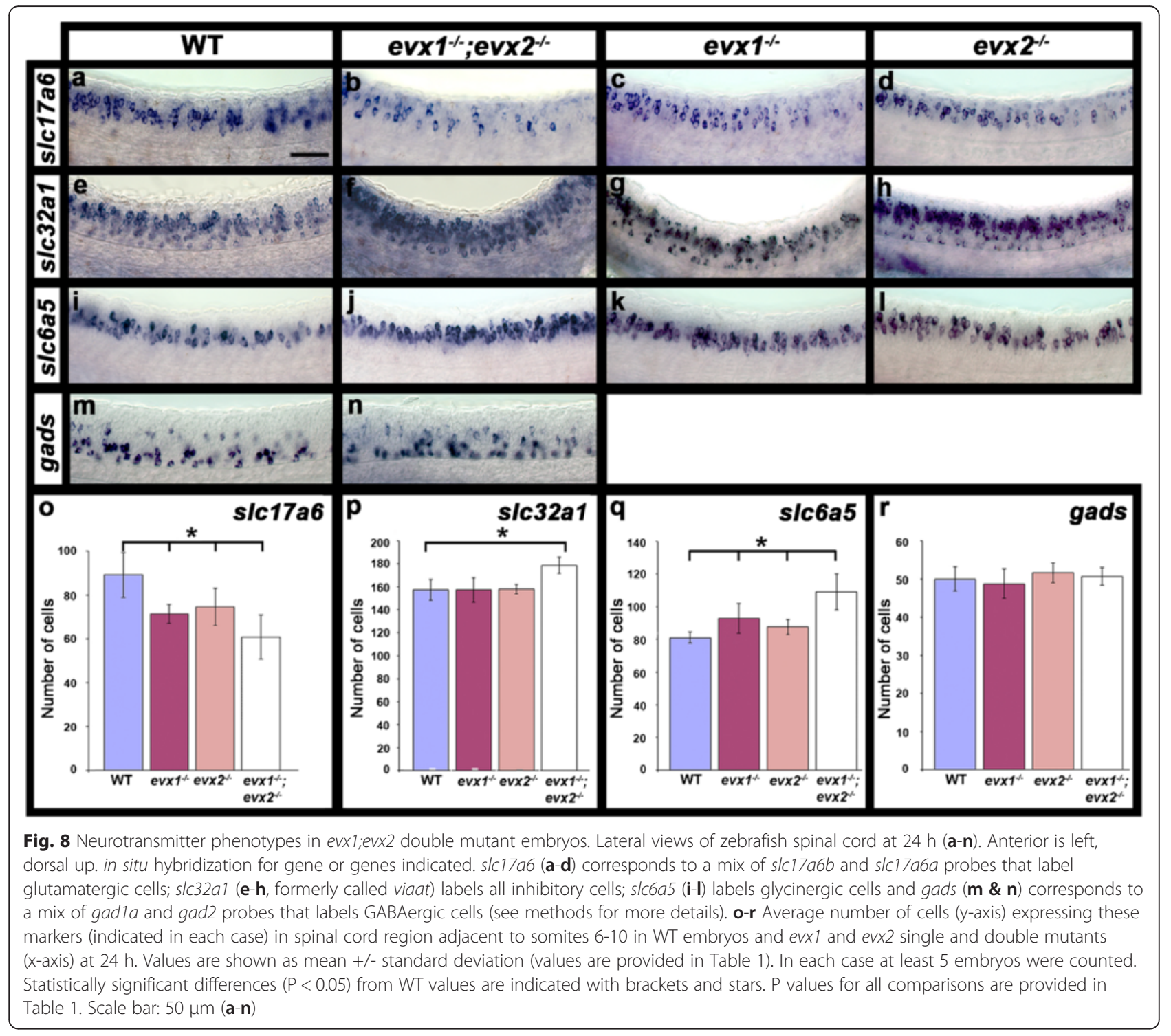

positions of these $e v x$ genes (adjacent to specific Hox clusters) along with phylogenetic analyses strongly suggest that the third $e v x$ gene in teleosts (eve1) is not the result of the extra genome duplication in the teleost lineage ([26, 78, 79], Additional file 1). Instead, it is likely that all three of these genes originated from the two rounds of whole genome duplication that occurred early in the vertebrate lineage [80] and eve1 was later lost in the tetrapod lineage ([26, 78], Additional file 1).

Here we provide the first comprehensive analysis of the functions of Evx1 and Evx2 in spinal cord interneuron development in any vertebrate. We demonstrate that, within the spinal cord, both of these transcription factors are expressed exclusively by V0v cells. We also show that V0v cells are glutamatergic. These findings complement and extend those of Satou and colleagues [36], who reported that Evx2-expressing cells that develop from the $d b x 1 b$ progenitor domain in zebrafish express the glutamateric maker slc17a6b and Talpalar and colleagues [9], who showed that mouse V0v cells express the glutamatergic marker slc17a6 (vglut2). In addition, we confirm and extend previous reports that suggested that V0v neurons extend commissural axons $[23,27,35,36]$ and we identify these neurons as CoSA interneurons. Interestingly, while Satou and colleagues [36] observed similar V0v cell morphologies at the stages that we have examined, at later stages of development they also saw descending and bifurcating commissural excitatory V0 cells, suggesting that V0v cells may diversify morphologically at later developmental stages [36].

In mouse Evx1 mutants, most V0v cells completely change their fate and acquire characteristics of V1 cells, the cell-type that normally forms ventral to V0v cells. Cells that would have formed V0v interneurons lose 
expression of Evx1 and Evx2 and instead express the V1 marker Engrailed1 (En1) and develop axon trajectories and migration patterns characteristic of $\mathrm{V} 1$ interneurons [23]. Most notably, their axon trajectories change from being contralateral to ipsilateral [23]. In addition, experiments in chick embryos revealed that ectopic Evx is sufficient to suppress Engrailed expression and therefore presumably V1 cell fate [23]. The role of Evx2 in mouse V0v cells is less well understood. Evx2 expression is dependent on Evx1, suggesting that it may be involved in the specification events described above [23], but spinal cord phenotypes of $E v x 2$ mutants have not been described in mouse and before this study, Evx1;Evx2 double mutants had not been described in any vertebrate.

These amniote data suggest that Evx1 is required to inhibit the V1 fate in post-mitotic V0v cells. This global cell fate change is unusual for a transcription factor expressed in post-mitotic cells: it is more commonly seen with transcription factors expressed in spinal progenitor domains (e.g. [27-29, 81, 82]). For example, Nkx2.2 is a transcription factor expressed in the p3 progenitor domain and in $N k \times 2.2$ mutant mice, cells that would have formed V3 interneurons change their fates (transfate) and become motoneurons instead [83]. Similarly, Dbx1 is expressed in the progenitor domain (p0) from which V0 cells develop and in $D b x 1$ mutant mice, cells that would have become V0v cells assume the characteristics of V1 cells [27, 28].

Interestingly, our results are different and yield novel insights into Evx1 and Evx2 function in the spinal cord. We see no evidence of V0v cells transfating to V1 cells in zebrafish evx1 and evx2 single or double mutants. Notably, there is no increase in the number of cells expressing eng 1 , which is specifically expressed in V1 cells, or Pax2, which is expressed by $\mathrm{V} 1$ and $\mathrm{V}_{\mathrm{D}}$ cells. Instead most $\mathrm{V} 0 \mathrm{v}$ cells continue to express evx1 mRNA and $\operatorname{Tg}($ evx1:EGFP) and these EGFP-labelled V0v neurons have what appear to be normal CoSA axon morphologies. These data strongly suggest that V0v cells still form in zebrafish evx1;evx2 double mutants and that they do not become a different class of neuron. One possible explanation for the differences between our results and the previously reported analyses in mouse might be evolutionary changes in the functions of Evx1 and Evx2. However, it is also possible that the consequences of removing these transcription factors are different in mouse and zebrafish because of variations in the expression of Dbx1 and/or the timing of V0v development. Interestingly, in this paper, we have shown that expression of $d b x 1 a$ and $d b \times 1 b$ persists in at least some V0v cells in zebrafish. Therefore, it is possible that in zebrafish evx1;evx2 mutants, Dbx might be able to inhibit post-mitotic V0v cells from becoming V1 cells
(Fig. 9). Given the speed of zebrafish spinal cord development it is also possible that V0v cells become committed to their fate faster than in mouse and that, therefore, the window of time during which the V1 fate needs to be inhibited in V0v cells is much shorter in zebrafish than in mammals (see [84] for a different example of how the fast speed of zebrafish development can produce changes in spinal cord development). Regardless of how V0v global cell fate specification has evolved, the fact that V0v cells still form in zebrafish lacking Evx1 and Evx2, has provided us with a unique opportunity to identify Evx functions in V0v cells, independent of any role that these transcription factors may also have in repressing the V1 cell fate.

Our results show that in zebrafish, Evx1 and Evx2 act partially redundantly to specify the glutamatergic fate of V0v cells and inhibit an alternative glycinergic fate in these cells. Given that the only spinal cord cells that express evx1 and evx2 are V0v cells and that the only other trunk tissue that expresses either of these genes is the posterior gut, which expresses $e v x 1$, we consider that this requirement for $\operatorname{Evx} 1$ and Evx2 function is likely to be cell-autonomous. Interestingly, while there is a reduction of glutamatergic cells in both single and double mutants, expression of the inhibitory marker slc32a1 is only increased in double mutants, suggesting that the specification of glutamatergic fates and the inhibition of glycinergic fates may be independent processes which require different levels of Evx activity. However, in contrast to slc32a1, the number of cells expressing the glycinergic marker slc6a5 was slightly increased in single mutants, which suggests that the expression of different neurotransmitter transporter proteins is regulated independently and by distinct levels of Evx activity. These results are intriguing as they suggest that the regulation of neurotransmitter transporters and enzymes might be complex, with different components being regulated by distinct mechanisms.

V0v interneurons are a crucial part of locomotor circuitry as they are required for hindlimb left-right alternation during fast locomotion [9, 27, 34]. Therefore, changing the neurotransmitter fate of these cells might be expected to impair fast movements. Unfortunately, as evx2 mutants die by larval stages, we were not able to assess whether evx2 single mutants or evx1;evx2 double mutants have locomotion defects. In addition, evxl single mutants lack joints in their fins [39], making it impossible to evaluate if any difference in evx1 single mutant behavior is due to this fin phenotype or a locomotive defect. Interestingly, we did not observe any obvious changes in V0v cell morphology or axon trajectory in evx1;evx2 double mutants. Given the changed neurotransmitter 


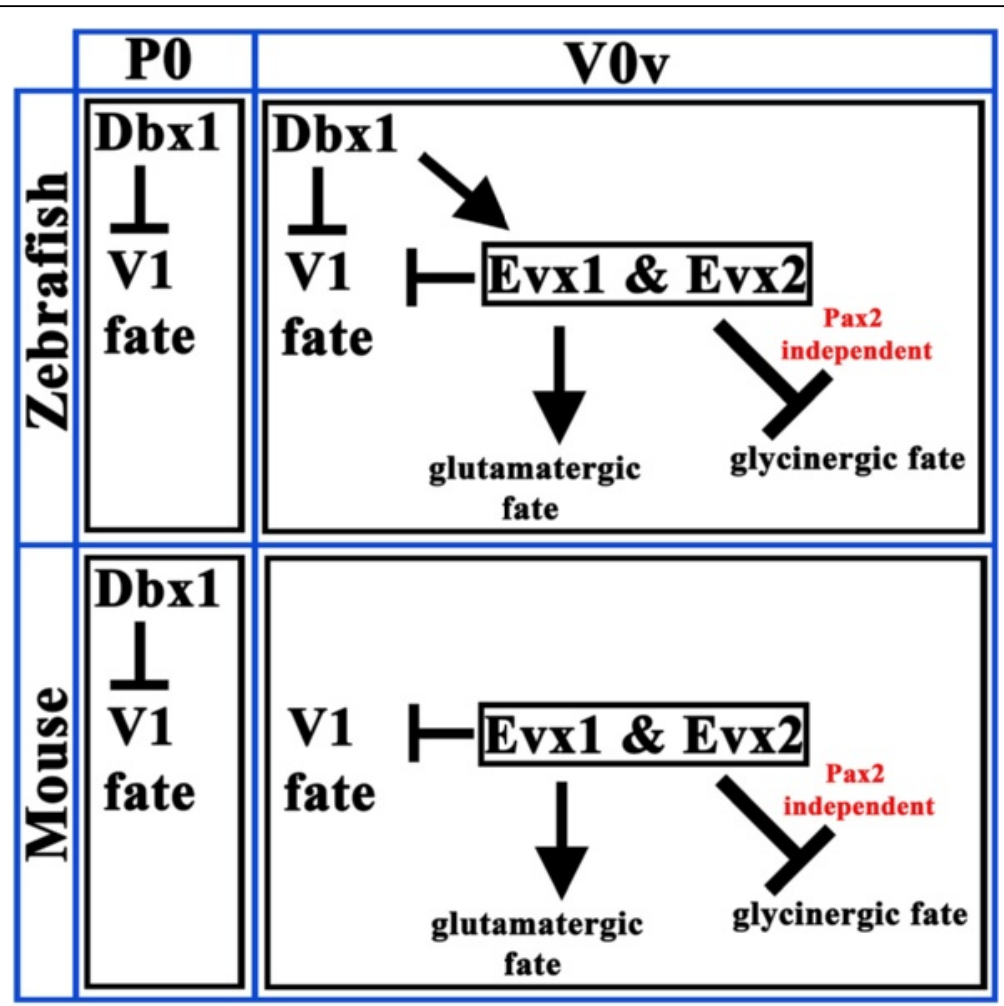

Fig. 9 A Model for Evx Function in Vov cells. A possible model that would reconcile the different phenotypes of mouse and zebrafish Evx 1 mutants. In this model, Dbx1 expression in P0 cells is required for the expression of Evx1 and Evx2 in V0v cells (for simplicity this interaction is not shown) and Dbx1 can also independently repress the V1 fate. In zebrafish, Dbx1 expression persists for a while in newly formed Vov cells. This may be sufficient for the $\mathrm{V} 1$ fate to be inhibited in post-mitotic Vov cells, even in the absence of Evx 1 and Evx2, thereby revealing other functions of Evx transcription factors. This could explain why Vov cells still form in evx1;evx2 double mutants, but they express glycinergic rather than glutamatergic markers. In contrast, in mouse Dbx1 is expressed only in progenitor cells. Therefore, in newly formed V0v cells only Evx1 and Evx2 inhibit the V1 fate and in the absence of these transcription factors V0v cells transfate into V1 cells

phenotype of V0v cells in these animals this might be considered surprising, although it is consistent with our previous analysis of V1 cells, that maintain their ipsilateral axon trajectories even when they lose their inhibitory fates in the absence of Pax2 and Pax8 [14]. It is still possible though that there are subtle changes in V0v cell wiring and/or changes in V0v cell connectivity in evx1;evx2 double mutants as a result of their change in neurotransmitter fate. As there are fewer GFP-labelled V0v cells in evx1;evx2 double mutants it is also possible that VOv cells with inappropriate neurotransmitter fates eventually die, although alternatively this reduction in the number of GFPpositive cells may just reflect autoregulation of Evx expression.

In this paper, we also describe the expression of a different transcription factor gene expressed by V0v cells, skor2. Skor2 expression has also been reported in the mouse spinal cord but the cells that express it were not identified [85]. Our results show that skor2 is expressed by a subset of $\mathrm{VO} \mathrm{v}$ cells as well as at least one population of more dorsal excitatory spinal cord cells.
We also demonstrate that expression of skor 2 in V0v cells requires Evx1 and Evx2 activity. Given that skor2 is predominantly expressed by excitatory cells, it is possible that it acts downstream of Evx1 and Evx2 in V0v cells in either the specification of glutamatergic fates and/or the inhibition of glycinergic fates and that it might also have this function in other cells. However it is also possible that Skor2 acts downstream of Evx1 and Evx2 in some other as-yet-unidentified aspect of V0v cell specification. These alternatives can be tested by future loss-of-function analyses of Skor2.

Excitingly, in addition to demonstrating the roles of Evx1 and Evx2 in neurotransmitter specification, our data also show that these transcription factors function independently of Pax2 in specifying glutamatergic fates and inhibiting glycinergic fates. This is the first time that a Pax2-independent mechanism of inhibitory fate specification has been identified in the spinal cord. While several transcription factors have been identified that specify the inhibitory fates of particular spinal cord neurons, so far all of these act upstream of Pax2 [14-18]. In addition, as mentioned before, the only other transcription factors that 
have been identified as specifying excitatory spinal cord fates, Tlx1 and Tlx3 $[4,5,8]$, work at least in part by down-regulating Pax2 [4]. Therefore, our finding that V0v cells become inhibitory in evx1;evx2 double mutants but do not express Pax2 is a significant one as it demonstrates that there must be an additional Pax2-independent mechanism for specifying inhibitory neurons in the spinal cord.

\section{Conclusions}

In conclusion, in this paper we demonstrate that zebrafish V0v cells express $e v x 1$ and $e v x 2$ and develop into excitatory (glutamatergic) CoSA interneurons. We also show that Evx1 and Evx2 are required, partially redundantly for expression of skor 2 and glutamatergic markers and inhibition of glycinergic markers in V0v cells and that in the absence of Evx1 and Evx2 function V0v cells become glycinergic through a novel Pax2-independent mechanism. Taken together, our data significantly increase our understanding of how neurotransmitter fates are specified and the genetic networks involved in these processes.

\section{Additional file}

Additional file 1: Supplementary Data. (PDF $2.67 \mathrm{mb}$ )

\section{Abbreviations}

CNE: conserved non-coding element; CNS: central nervous system; CoSA: commisural secondary ascending; DAB: 3,3'- diaminobenzidine; dpf: days post fertilization; FACS: fluorescent activated cell sorting; Gads: glutamic acid decarboxylases; h: hours post fertilization; LHS: left hand side; RHS: right hand side; WT: wild type.

\section{Competing interests}

The authors have no competing interests.

\section{Authors' contributions}

JM created the Tg(evx:EGFP) lines and performed most of the experiments in the paper, including most of the single and double labeling experiments in WT, single mutant and double mutant embryos and all of the cell counts except those for the skor2 double labeling experiments; CS performed initial experiments with the evx 1 mutant and evx 1 and evx2 morpholinos and helped to formulate many of the hypotheses that this study tests; CS also performed many of the WT expression analyses of evx1, evx2 and eve1, including some of the initial double labeling experiments; SE performed the synteny and phylogenetic analyses; SP and GV genotyped embryos and adult fish and helped to establish stable $T g$ (evx:EGFP) WT and mutant lines; SP and GV also examined Evx2 expression in WT and evx2 single mutant embryos and performed the evx2 mutant survival experiments; SP also performed the double staining for Evx2 and EGFP in Tg(s/c17a6:EGFP) embryos; WH performed the initial analysis of skor2 and the double labeling experiments between skor2 and other markers; SE and SdJ performed the FACS and expression profiling analyses; $\mathrm{KL}$ designed and directed the study; $\mathrm{KL}$ and JM wrote most of the paper with input from the other authors.

\section{Acknowledgments}

We thank Dr Shinichi Higashijima for kindly providing us with Evx2 antibody, Dr Derek Stemple and everyone working on the zebrafish mutation project at the Wellcome Trust Sanger Centre for the evx2 mutant allele, ZFIN for providing information on nomenclature and other essential zebrafish resources, Nigel Miller at the Flow Cytometry Facility, Department of Pathology, University of Cambridge, Cambridge, for his expert FAC-sorting, Tomi Ivacevic and Vladimir Benes at the Genomics Core Facility at EMBL, Heidelberg, for RNA amplification, labelling, and hybridization, Uwe Strähle,
Olivier Armant and Jasmin Lampert for help with designing microarrays, Nicole Santos for help with genotyping, Henry Putz, Jessica Bouchard, Annika Swanson and several SU undergraduate fish husbandry workers for help with maintaining zebrafish lines and Dr Santanu Banerjee for helpful comments on a previous version of this manuscript.

\section{Funding}

This work was supported by NSF IOS-1257583, NIH NINDS R21NS073979, the Spinal Cord Injury Trust Fund through New York State Department of Health Contract \#C030177, a Royal Society University Research Fellowship and Syracuse University start-up funds awarded to K.E.L; BBSRC, Cambridge Trust, DAAD and Daimler-Benz-Foundation funding awarded to C.J.S; NSF HRD0703452 LSAMP and Syracuse University Ruth Meyer funding awarded to S.A.P and NSF HRD-1202480 LSAMP and Syracuse University Ruth Meyer funding awarded to G.K.V.

\section{Author details}

${ }^{1}$ Department of Biology, Syracuse University, 107 College Place, Syracuse, NY 13244, USA. 'Department of Physiology, Development and Neuroscience, University of Cambridge, Downing Street, Cambridge CB2 3DY, UK. ${ }^{3}$ Department of Neuroscience and Physiology, SUNY Upstate Medical University, 505 Irving Avenue, Syracuse, NY 13210, USA.

Received: 14 December 2015 Accepted: 4 February 2016

Published online: 19 February 2016

\section{References}

1. Goulding M. Circuits controlling vertebrate locomotion: moving in a new direction. Nat Rev Neurosci. 2009;10(7):507-18.

2. Lewis KE. How do genes regulate simple behaviours? Understanding how different neurons in the vertebrate spinal cord are genetically specified. Philos Trans R Soc Lond B Biol Sci. 2006;361(1465):45-66.

3. Gosgnach S, Lanuza GM, Butt SJ, Saueressig H, Zhang Y, Velasquez T, et al. V1 spinal neurons regulate the speed of vertebrate locomotor outputs. Nature. 2006;440(7081):215-9.

4. Li WC, Higashijima S, Parry DM, Roberts A, Soffe SR. Primitive roles for inhibitory interneurons in developing frog spinal cord. J Neurosci. 2004; 24(25):5840-8.

5. Higashijima S, Masino M, Mandel G, Fetcho JR. Engrailed-1 Expression Marks a Primitive Class of Inhibitory Spinal Interneuron. J Neurosci. 2004;24(25): 5827-39.

6. Crone SA, Zhong G, Harris-Warrick R, Sharma K. In mice lacking V2a interneurons, gait depends on speed of locomotion. J Neurosci. 2009;29(21): 7098-109.

7. Crone SA, Quinlan KA, Zagoraiou L, Droho S, Restrepo CE, Lundfald L, et al. Genetic ablation of V2a ipsilateral interneurons disrupts left-right locomotor coordination in mammalian spinal cord. Neuron. 2008;60(1):70-83.

8. Kimura Y, Okamura Y, Higashijima S. alx, a zebrafish homolog of Chx10, marks ipsilateral descending excitatory interneurons that participate in the regulation of spinal locomotor circuits. J Neurosci. 2006;26(21):5684-97.

9. Talpalar AE, Bouvier J, Borgius L, Fortin G, Pierani A, Kiehn O. Dual-mode operation of neuronal networks involved in left-right alternation. Nature. 2013;500(7460):85-8

10. Xue M, Atallah BV, Scanziani M. Equalizing excitation-inhibition ratios across visual cortical neurons. Nature. 2014;511(7511):596-600

11. Zadori D, Veres G, Szalardy L, Klivenyi P, Toldi J, Vecsei L. Glutamatergic dysfunctioning in Alzheimer's disease and related therapeutic targets. J Alzheimers Dis. 2014:42 Suppl 3:S177-187.

12. Bateup HS, Johnson CA, Denefrio CL, Saulnier JL, Kornacker K, Sabatini BL. Excitatory/inhibitory synaptic imbalance leads to hippocampal hyperexcitability in mouse models of tuberous sclerosis. Neuron. 2013; 78(3):510-22

13. Pittenger $\mathrm{C}$, Bloch MH, Williams K. Glutamate abnormalities in obsessive compulsive disorder: neurobiology, pathophysiology, and treatment. Pharmacol Ther. 2011;132(3):314-32.

14. Batista MF, Lewis KE. Pax2/8 act redundantly to specify glycinergic and GABAergic fates of multiple spinal interneurons. Dev Biol. 2008;323(1):88-97.

15. Cheng L, Samad OA, Xu Y, Mizuguchi R, Luo P, Shirasawa S, et al. Lbx 1 and Tlx3 are opposing switches in determining GABAergic versus glutamatergic transmitter phenotypes. Nat Neurosci. 2005;8(11):1510-5. 
16. Mizuguchi R, Kriks S, Cordes R, Gossler A, Ma Q, Goulding M. Ascl1 and Gsh1/2 control inhibitory and excitatory cell fate in spinal sensory interneurons. Nat Neurosci. 2006;9(6):770-8.

17. Glasgow SM, Henke RM, Macdonald RJ, Wright CV, Johnson JE. Ptf1a determines GABAergic over glutamatergic neuronal cell fate in the spinal cord dorsal horn. Development. 2005;132(24):5461-9.

18. Pillai A, Mansouri A, Behringer R, Westphal H, Goulding M. Lhx1 and Lhx5 maintain the inhibitory-neurotransmitter status of interneurons in the dorsal spinal cord. Development. 2007;134(2):357-66.

19. Guo Z, Zhao C, Huang M, Huang T, Fan M, Xie Z, et al. Tlx1/3 and Ptf1a control the expression of distinct sets of transmitter and peptide receptor genes in the developing dorsal spinal cord. J Neurosci. 2012;32(25):8509-20.

20. Huang M, Huang T, Xiang Y, Xie Z, Chen Y, Yan R, et al. Ptf1a, Lbx1 and Pax2 coordinate glycinergic and peptidergic transmitter phenotypes in dorsal spinal inhibitory neurons. Dev Biol. 2008;322(2):394-405.

21. Hori K, Cholewa-Waclaw J, Nakada Y, Glasgow SM, Masui T, Henke RM, et al. A nonclassical bHLH Rbpj transcription factor complex is required for specification of GABAergic neurons independent of Notch signaling. Gene Dev. 2008;22(2):166-78.

22. Cheng L, Arata A, Mizuguchi R, Qian Y, Karunaratne A, Gray PA, et al. Tlx3 and $\mathrm{T} \mid \mathrm{X} 1$ are post-mitotic selector genes determining glutamatergic over GABAergic cell fates. Nat Neurosci. 2004;7(5):510-7.

23. Moran-Rivard L, Kagawa T, Saueressig H, Gross MK, Burrill J, Goulding M. Evx 1 is a postmitotic determinant of V0 interneuron identity in the spinal cord. Neuron. 2001;29(2):385-99.

24. Dolle P, Fraulob V, Duboule D. Developmental expression of the mouse Evx-2 gene: relationship with the evolution of the HOM/Hox complex. Dev Suppl. 1994;143-153.

25. Bastian H, Gruss P. A murine even-skipped homologue, Evx 1, is expressed during early embryogenesis and neurogenesis in a biphasic manner. EMBO J. 1990;9(6):1839-52.

26. Avaron F, Thaeron-Antono C, Beck CW, Borday-Birraux V, Geraudie J, Casane D, et al. Comparison of even-skipped related gene expression pattern in vertebrates shows an association between expression domain loss and modification of selective constraints on sequences. Evol Dev. 2003;5(2):145-56.

27. Lanuza G, Gosgnach S, Pierani A, Jessel T, Goulding M. Genetic Identification of Spinal Interneurons that Coordinate Left-Right Locomotor Activity Necessary for Walking Movements. Neuron. 2004:42:375-86.

28. Pierani A, Moran-Rivard L, Sunshine MJ, Littman DR, Goulding M, Jessell TM. Control of interneuron fate in the developing spinal cord by the progenitor homeodomain protein Dbx1. Neuron. 2001;29(2):367-84.

29. Briscoe J, Pierani A, Jessell TM, Ericson J. A homeodomain protein code specifies progenitor cell identity and neuronal fate in the ventral neural tube. Cell. 2000;101(4):435-45.

30. Ruiz i Altaba A, Melton DA. Bimodal and graded expression of the Xenopus homeobox gene Xhox3 during embryonic development. Development. 1989;106(1):173-83.

31. Ruiz i Altaba A. Neural expression of the Xenopus homeobox gene Xhox3: evidence for a patterning neural signal that spreads through the ectoderm. Development. 1990;108(4):595-604.

32. Sordino P, Duboule D, Kondo T. Zebrafish Hoxa and Evx-2 genes: cloning, developmental expression and implications for the functional evolution of posterior Hox genes. Mech Dev. 1996;59:165-75.

33. Thaeron C, Avaron F, Casane D, Borday V, Thisse B, Thisse C, et al. Zebrafish evx1 is dynamically expressed during embryogenesis in subsets of interneurones, posterior gut and urogenital system. Mech Dev. 2000;99:167-72.

34. Griener A, Zhang W, Kao H, Wagner C, Gosgnach S. Probing diversity within subpopulations of locomotor-related V0 interneurons. Developmental neurobiology. 2015.

35. Suster ML, Kania A, Liao M, Asakawa K, Charron F, Kawakami K, et al. A novel conserved evx1 enhancer links spinal interneuron morphology and cisregulation from fish to mammals. Dev Biol. 2009;325(2):422-33.

36. Satou C, Kimura Y, Higashijima S. Generation of multiple classes of V0 neurons in zebrafish spinal cord: progenitor heterogeneity and temporal control of neuronal diversity. J Neurosci. 2012;32(5):1771-83.

37. Bae YK, Kani S, Shimizu T, Tanabe K, Nojima H, Kimura Y, et al. Anatomy of zebrafish cerebellum and screen for mutations affecting its development. Dev Biol. 2009;330(2):406-26.

38. Kimmel CB, Ballard WW, Kimmel SR, Ullmann B, Schilling TF. Stages of embryonic development of the zebrafish. Dev Dyn. 1995;203(3):253-310.
39. Schulte CJ, Allen C, England SJ, Juarez-Morales JL, Lewis KE. Evx1 is required for joint formation in zebrafish fin dermoskeleton. Dev Dyn. 2011;240(5):1240-8.

40. Brudno M, Do CB, Cooper GM, Kim MF, Davydov E, Green ED, et al. LAGAN and Multi-LAGAN: efficient tools for large-scale multiple alignment of genomic DNA. Genome Res. 2003;13(4):721-31.

41. Mayor C, Brudno M, Schwartz JR, Poliakov A, Rubin EM, Frazer KA, et al. VISTA : visualizing global DNA sequence alignments of arbitrary length. Bioinformatics. 2000;16(11):1046-7.

42. Sasaki Y, Sone T, Yoshida S, Yahata K, Hotta J, Chesnut JD, et al. Evidence for high specificity and efficiency of multiple recombination signals in mixed DNA cloning by the Multisite Gateway system. J Biotechnol. 2004;107(3):233-43.

43. Suzuki Y, Kagawa N, Fujino T, Sumiya T, Andoh T, Ishikawa K, et al. A novel high-throughput (HTP) cloning strategy for site-directed designed chimeragenesis and mutation using the Gateway cloning system. Nucleic Acids Res. 2005;33(12):1-6.

44. Villefranc JA, Amigo J, Lawson ND. Gateway compatible vectors for analysis of gene function in the zebrafish. Dev Dyn. 2007;236(11):3077-87.

45. Köster RW, Fraser SE. Tracing transgene expression in living zebrafish embryos. Dev Biol. 2001;233(2):329-46.

46. Wang Y, Shen J, Arenzana N, Tirasophon W, Kaufman RJ, Prywes R. Activation of ATF6 and an ATF6 DNA binding site by the endoplasmic reticulum stress response. J Biol Chem. 2000;275(35):27013-20.

47. Kawakami K, Takeda H, Kawakami N, Kobayashi M, Matsuda N, Mishina M. A transposon-mediated gene trap approach identifies developmentally regulated genes in zebrafish. Dev Cell. 2004;7(1):133-44.

48. Halpern ME, Rhee J, Goll MG, Akitake CM, Parsons M, Leach SD. Gal4/UAS transgenic tools and their application to zebrafish. Zebrafish. 2008;5(2):97-110.

49. Cerda GA, Hargrave M, Lewis KE. RNA profiling of FAC-sorted neurons from the developing zebrafish spinal cord. Dev Dyn. 2009;238(1):150-61.

50. England S, Hilinski W, de Jager S, Andrzejczuk L, Campbell P, Chowdhury T, Demby C, Fancher W, Gong Y, Lin C et al: Identifying Transcription Factors expressed by Ventral Spinal Cord Interneurons. ZFIN on-line publication 2014, http://zfin.org/ZDB-PUB-140822-10.

51. Park HC, Kim CH, Bae YK, Yeo SY, Kim SH, Hong SK, et al. Analysis of upstream elements in the HuC promoter leads to the establishment of transgenic zebrafish with fluorescent neurons. Dev Biol. 2000;227(2):279-93.

52. Picker A, Scholpp S, Bohli H, Takeda H, Brand M. A novel positive transcriptional feedback loop in midbrain-hindbrain boundary development is revealed through analysis of the zebrafish pax2.1 promoter in transgenic lines. Development. 2002;129:3227-39.

53. Peri F, Nusslein-Volhard C. Live imaging of neuronal degradation by microglia reveals a role for v0-ATPase a1 in phagosomal fusion in vivo. Cell. 2008;133(5):916-27.

54. Kobayashi M, Nishikawa K, Yamamoto M. Hematopoietic regulatory domain of gata1 gene is positively regulated by GATA1 protein in zebrafish embryos. Development. 2001;128(12):2341-50.

55. Batista MF, Jacobstein J, Lewis KE. Zebrafish V2 cells develop into excitatory $\mathrm{CiD}$ and Notch signalling dependent inhibitory VeLD interneurons. Dev Biol. 2008;322(2):263-75.

56. Concordet JP, Lewis KE, Moore JW, Goodrich LV, Johnson RL, Scott MP, et al. Spatial regulation of a zebrafish patched homologue reflects the roles of sonic hedgehog and protein kinase $A$ in neural tube and somite patterning. Development. 1996;122(9):2835-46.

57. Gribble SL, Nikolaus OB, Dorsky RI. Regulation and function of Dbx genes in the zebrafish spinal cord. Dev Dyn. 2007;236(12):3472-83.

58. Thaëron C, Avaron F, Casane D, Borday V, Thisse B, Thisse C, et al. Zebrafish evx1 is dynamically expressed during embryogenesis in subsets of interneurones, posterior gut and urogenital system. Mech Dev. 2000;99(1-2):167-72.

59. Joly JS, Joly C, Schulte-Merker S, Boulekbache H, Condamine H. The ventral and posterior expression of the zebrafish homeobox gene eve1 is perturbed in dorsalized and mutant embryos. Development. 1993;119(4):1261-75.

60. Pfeffer PL, Gerster T, Lun K, Brand M, Busslinger M. Characterization of three novel members of the zebrafish Pax2/5/8 family: dependency of Pax 5 and Pax8 expression on the Pax2.1 (noi) function. Development. 1998;125(16):3063-74.

61. Higashijima S, Mandel G, Fetcho JR. Distribution of prospective glutamatergic, glycinergic, and GABAergic neurons in embryonic and larval zebrafish. J Comp Neurol. 2004;480(1):1-18.

62. Higashijima S, Schaefer M, Fetcho JR. Neurotransmitter properties of spinal interneurons in embryonic and larval zebrafish. J Comp Neurol. 2004;480(1):19-37. 
63. Pierani A, Brenner-Morton S, Chiang C, Jessell TM. A Sonic Hedgehog Independent Retinoid-Activated Pathway of Neurogenesis in the Ventral Spinal Cord. Cell. 1999;97:903-15.

64. Cruz C, Maegawa S, Weinberg ES, Wilson SW, Dawid IB, Kudoh T. Induction and patterning of trunk and tail neural ectoderm by the homeobox gene eve1 in zebrafish embryos. Proc Natl Acad Sci U S A. 2010;107(8):3564-9.

65. Lewis KE, Eisen JS. From cells to circuits: development of the zebrafish spinal cord. Prog Neurobiol. 2003;69(6):419-49.

66. Bernhardt RR, Chitnis AB, Lindamer L, Kuwada JY. Identification of spinal neurons in the embryonic and larval zebrafish. J Comp Neurol. 1990;302:603-16.

67. Satou C, Kimura Y, Hirata H, Suster ML, Kawakami K, Higashijima S. Transgenic tools to characterize neuronal properties of discrete populations of zebrafish neurons. Development. 2013;140(18):3927-31.

68. McIntire SL, Reimer RJ, Schuske K, Edwards RH, Jorgensen EM. Identification and characterization of the vesicular GABA transporter. Nature. 1997; 389(6653):870-6.

69. Sagne C, El Mestikawy S, Isambert MF, Hamon M, Henry JP, Giros B, et al. Cloning of a functional vesicular GABA and glycine transporter by screening of genome databases. FEBS letters. 1997;417(2):177-83.

70. Rabe N, Gezelius H, Vallstedt A, Memic F, Kullander K. Netrin-1-dependent spinal interneuron subtypes are required for the formation of left-right alternating locomotor circuitry. J Neurosci. 2009;29(50):15642-9.

71. Burrill JD, Moran L, Goulding MD, Saueressig H. PAX2 is expressed in multiple spinal cord interneurons, including a population of EN1+ interneurons that require PAX6 for their development. Development. 1997; 124(22):4493-503.

72. Ahringer J. Posterior patterning by the Caenorhabditis elegans even-skipped homolog vab-7. Gene Dev. 1996;10(9):1120-30

73. Briata P, llengo C, Van DeWerken R, Corte G. Mapping of a potent transcriptional repression region of the human homeodomain protein EVX1. FEBS letters. 1997;402(2-3):131-5.

74. Biggin MD, Tjian R. A purified Drosophila homeodomain protein represses transcription in vitro. Cell. 1989;58(3):433-40.

75. TenHarmsel A, Austin RJ, Savenelli N, Biggin MD. Cooperative binding at a distance by even-skipped protein correlates with repression and suggests a mechanism of silencing. Mol Cell Biol. 1993:13(5):2742-52.

76. Li C, Manley JL. Even-skipped represses transcription by binding TATA binding protein and blocking the TFIID-TATA box interaction. Mol Cell Biol. 1998;18(7):3771-81.

77. Han K, Manley JL. Functional domains of the Drosophila Engrailed protein. EMBO J. 1993;12:2723-33.

78. Amores A, Force A, Yan YL, Joly L, Amemiya C, Fritz A, et al. Zebrafish hox clusters and vertebrate genome evolution. Science. 1998;282(5394):1711-4.

79. Taylor JS, Braasch I, Frickey T, Meyer A, Van de Peer Y. Genome duplication, a trait shared by 22000 species of ray-finned fish. Genome Res. 2003;13(3): 382-90.

80. Holland PW, Garcia-Fernandez J, Williams NA, Sidow A. Gene duplications and the origins of vertebrate development. Dev Suppl. 1994;125-133.

81. Ericson J, Rashbass P, Schedl A, Brenner-Morton S, Kawakami A, van Heyningen $V$, et al. Pax6 controls progenitor cell identity and neuronal fate in response to graded Shh signaling. Cell. 1997;90:169-80.

82. Sander M, Paydar S, Ericson J, Briscoe J, Berber E, German M, et al. Ventral neural patterning by Nkx homeobox genes: Nkx6.1 controls somatic motor neuron and ventral interneuron fates. Gene Dev. 2000;14(17):2134-9.

83. Briscoe J, Sussel L, Serup P, Hartigan-O'Connor D, Jessell TM, Rubenstein JL, et al. Homeobox gene Nkx2.2 and specification of neuronal identity by graded Sonic hedgehog signalling. Nature. 1999;398(6728):622-7.

84. England S, Batista MF, Mich JK, Chen JK, Lewis KE. Roles of Hedgehog pathway components and retinoic acid signalling in specifying zebrafish ventral spinal cord neurons. Development. 2011:138(23):5121-34.

85. Arndt S, Poser I, Schubert T, Moser M, Bosserhoff AK. Cloning and functional characterization of a new Ski homolog, Fussel-18, specifically expressed in neuronal tissues. Lab Investig. 2005;85(11):1330-41.

\section{Submit your next manuscript to BioMed Central and we will help you at every step:}

- We accept pre-submission inquiries

- Our selector tool helps you to find the most relevant journal

- We provide round the clock customer support

- Convenient online submission

- Thorough peer review

- Inclusion in PubMed and all major indexing services

- Maximum visibility for your research

Submit your manuscript at www.biomedcentral.com/submit
Biomed Central 